\title{
A Distributed Game-Theoretic Demand Response with Multi-Class Appliance Control in Smart Grid
}

\author{
Milad Latifi ${ }^{\mathrm{a}}$, Amir Rastegarnia ${ }^{\mathrm{a}, *}$, Azam Khalili $^{\mathrm{a}}$, Vahid Vahidpour $^{\mathrm{a}}$, Saeid Sanei ${ }^{\mathrm{b}}$ \\ ${ }^{a}$ Department of Electrical Engineering, Malayer University, Malayer, 65719-95863, Iran \\ ${ }^{b}$ School of Science and Technology, Nottingham Trent University, Clifton Lane, Nottingham, U.K.
}

\begin{abstract}
We propose an event-triggered game-theoretic strategy for managing the power grids demand side, capable of responding to changes in consumer preferences or the price parameters coming from the wholesale market. The relationship between the retailer and the residential consumers is modeled as one-leader, N-follower Stackelberg game. We provide a detailed characterization of the household appliances to reflect the reality and improve the efficiency of the demand response (DR). Moreover, to consider all the appliances' essentials, the consumer's objective function is formulated as a mixed integer non-linear program (MINLP), which, unlike conventional procedures, is solved via an integrated method. The proposed method consists of a day-ahead stage, in which the DR problem is solved for the next scheduling horizon, and a real-time stage which runs repeatedly to tackle the change in the parameters and adapt to the new condition. For any change in the grid, the consumers use the estimated optimal parameters (given by the original objective function) and develop another Stackelberg game based solution to maximize the satisfaction level. Given the appliances of multi-class nature, the proposed method is shown to be very tractable for ancillary services and reducing the mismatch between the renewable power generation and the load demand.
\end{abstract}

Keywords: Branch-and-bound, demand response, sequential quadratic programing, smart grid, Stackelberg game.

\section{Introduction}

One of the most important challenges in DR programs is system balancing and tackling the uncertainty in the availability of resources. In this context, the uses of electrical energy storage devices and thermostatically controlled loads (TCLs) are highly regarded as potential solutions for reducing peak demand and other challenges to balance the system. In addition, emerging new renewable and distributed energy sources increases the uncertainty in the smart grid and volatility in energy production, as challenging problems to solve. Energy storage devices and robust adaptive energy consumption scheduling framework decrease the impact of these factors by smoothing out the fluctuation of the consumption curve and reducing the mismatch between the energy supply and demand $[1,2]$.

In recent years the DR has become one of the most attractive areas of research and activities in the field of smart grids $[3,4,5,6,7,8,9,10,11,12,13,14]$. In [15] an optimization model has been proposed to schedule the hourly energy consumption in response to hourly changes in the electricity prices. In [16] an automatic and optimal residential energy consumption scheduling framework has been investigated which attempts to achieve a desired

*corresponding author, Email: rastegarnia@malayeru.ac.ir 
trade-off between minimizing the electricity payment and the waiting time for the operation of each appliance in household. An efficient pricing method for the DR with a few information exchanges between the consumers and the utility companies is proposed by Samadi et al. [17]. They analytically modeled each consumer's preference and energy consumption pattern in the form of a utility function and proposed a Vickrey-Clarke-Groves (VCG) mechanism for maximizing the social welfare. Angeli et al. [4] proposed a dynamic demand management solution for scheduling the power consumption of smart thermostatic domestic refrigerators. In their paper, the operating temperature and the energy consumption of these appliances are modified dynamically, within a safe range, in response to main's frequency fluctuations such as sudden power plant outages. A game theoretic-based energy consumption scheduling framework based on the use of mixed integer programming (MIP) to schedule the energy consumption pattern of residential consumers is provided in [5]. This paper aims to incorporate integration of locally generated renewable energy in order to minimize its dependency on conventional energy and the consumption cost.

The authors of [6] present a comprehensive and general optimization-based home energy management controller in response to the dynamic price signals, incorporating several classes of domestic appliances including deferrable, curtailable, thermal, and critical ones. Focusing on the TCLs, Tindemans et al. [7] discussed a novel decentralized stochastic control for controlling a large collection of appliances. In [8], a multi-class appliance load scheduling is presented for managing the energy consumption of a residence. The proposed method controls the operation time and energy consumption level of five classes of appliances adapting to time-of-use pricing in order to maximize the overall net utility of the residence while satisfying its budget limit. A decentralized real-time information based demand response (also called demand-side management) for minimizing peak-to-average ratio (PAR) and cost of power usage is proposed in [9] based on the game theoretical approaches. A DR load following strategy for an interconnected source and load system utilizing traditional units and population of cooling TCLs to cope with the mismatched power caused by the load activities and the renewable power injection in real time is discussed in [11].

There are also several works in the literature providing Stackelberg game-based solutions for the DR problem. To deal with this DR problem in a network of multiple utility companies, Maharjan et al. formulated a Stackelberg game between utility companies and end-users to maximize the revenue of each utility company and the payoff of each user [18]. They developed a distributed algorithm which converges to the equilibrium with only local information available for both utility companies and end-users. They also studied the impact of an attacker who can manipulate the price information from the utility companies and provided a scheme based on the concept of shared reserve power to improve the grid reliability and ensure its dependability. In [10], a real-time price based DR algorithm for achieving optimal load control of various devices in an appliance is proposed by forming a virtual electricity-trading process using one-leader, N-follower Stackelberg game.

A fully distributed algorithm that is able to optimize the aggregate cost, utility, and retailer's profit using the heuristic Diffusion-Stackelberg algorithm was presented in [19]. In this work, the interactions between consumers and retailer was modeled by the Stackelberg game and the adaptive diffusion algorithm was used to solve the consumers objective functions. A novel real-time pricing algorithm for the DR problem based on Stackelberg game was developed in [20] to minimize the consumers' bills and maximize the providers' profit. It defines the consumers' energy consumption and the providers' price by taking advantage of the bidirectional communication infrastructure while different producer-consumers (i.e. prosumers) negotiate with different energy providers. The authors of [21] 
presented a light-weight DR scheme based on the Stackelberg game model to reduce the communication overheads and delays. The proposed scheme manages energy consumption based on a non-iterative Stackelberg model and historical real-time pricing. In [22], Shinde and Swarup studied a general DR approach combining the behavior of electric vehicle users and residential customers with other elastic loads participating in the DR and considering different utility functions for different types of customers. Different cases with single and multiple utility companies (UCs) were considered in this work, trying to set the prices in such a way so as to maximize their profits. A Stackelberg game model was designed to address the conflict of interests between the UCs and the customers.

However, none of the mentioned works in the literature has provided a comprehensive DR solution with the ability to consider detailed model for all classes of residential devices, to adaptively track the drifts in location of the optimal parameters in real-time, and be implementable in a fully autonomous manner. Here, for the first time, we propose an event-triggered Stackelberg game-based distributed adaptive framework considering the storage devices, renewable sources, and detailed model for all class of appliances which gives many options to the consumers and retailer to reschedule the DR program in advance. The main contributions of this paper are therefore as follows:

Provision of an event-triggered framework: The presented approach is adaptive and is able to track the drifts in location of the optimal parameters. These drifts can result from the presence of unforeseen events, e.g., changes in price and renewable power production information, or in the consumer's preferences and objective function.

Multi-objective optimization: The proposed mechanism considers not only the consumers payment but also their satisfaction as well as maximization of the retailer profit, whilst the details on individual appliances are not required to be exchanged with those of neither retailer nor consumers.

An integrated approach: The algorithm which we use to solve the MINLP problem doesn't involve any decomposition technique. This scheme is based on branch-and-bound in which the branching is allowed after each iteration of solving non-linear part of the problem.

Fully classifying of the residential appliances: Due to considering all classes of residential appliances, our framework is very tractable to adapt with different tasks such as frequency regulation and load curve flattening.

The rest of this paper is organized as follows. In Section 2 we present the system model. In Section 3 we formulate the DR problem, and present our game theoretic strategy in 4 . Section 5 presents the experimental evaluation and in Section 6 we conclude the paper.

\section{System Model}

We consider a residential power system which consists of a service provider (retailer) and several consumers in a neighborhood. The service provider buys electricity from the wholesale market and sells it to the consumers. We assume that every consumer is equipped with smart meter and schedule his appliances with an automatic energy consumption scheduler (ECS) embedded in the smart meter, as depicted in Fig. 1. We further assume that communications between the wholesale market, the retailer and the consumers' smart meters are established by appropriate two-way communication protocols. We also consider multiple renewable sources e.g., a rooftop PV for consumers and a wind farm for retailer, that can provide a part of total energy demand of their consumers. For

each appliance, the consumers also need to set the admissible scheduling window $\mathcal{H}_{k, a} \triangleq\left\{\alpha_{k, a}, 1+\alpha \alpha_{k, a}, \cdots, \beta_{k, a}\right\} \in$ 


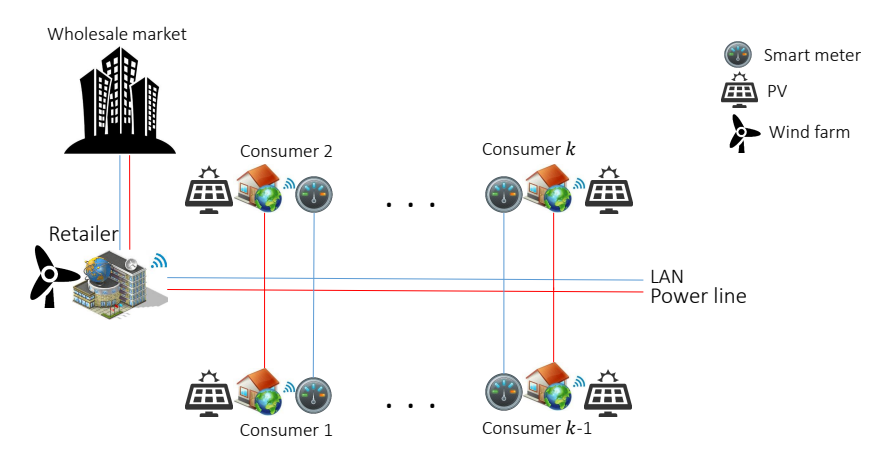

Figure 1: Block diagram of the smart micro-grid system.

$\mathcal{H} \triangleq\{1,2, \cdots, H\}$ by specifying the beginning and end of scheduling time. Here $\alpha_{k, a}$ represents minimum start time of operating range determined by consumer $k$ for appliance $a$. Similarly, $\beta_{k, a}\left(\alpha_{k, a}<\beta_{k, a}\right)$ represents the maximum stopping time of operating window before that, the device must complete its work.

\subsection{Classification of Appliances}

(I) Inelastic loads: These devices are not customizable and whenever necessary, should start work immediately, but the time of use of these appliances is not known in advance and have a stochastic on-off behavior with time varying properties. We show the set of these appliances for consumer $k$ as $\mathcal{A}_{k}^{I}$. TV and PC are two types of these devices. The behavior of these appliances is modeled as a discrete time Markov chain [23], with random integer decision variable $\boldsymbol{\kappa}_{k, a}^{h} \in\{0$ (off), 1 (on) $\}$ representing the state of the appliance $a \in \mathcal{A}_{k}^{I}$ at slot $h$ with the state probability row vector $P_{k, a}^{h}=\left(p_{k, a}^{h, f}, p_{k, a}^{h, o}\right)=\left(\operatorname{Pr}\left\{\boldsymbol{\kappa}_{k, a}^{h}=0\right\}, \operatorname{Pr}\left\{\boldsymbol{\kappa}_{k, a}^{h}=1\right\}\right) \cdot p_{k, a}^{h, f}$ (off-mode), $p_{k, a}^{h, o}$ (on-mode) are the probabilities of no change in the operational status, and $p_{k, a}^{h, f o}, p_{k, a}^{h, o f}$ are time varying transition probabilities from state off to state on and vice versa, respectively. The dynamic evolution in time of the state probability $P_{k, a}^{h+1}$ and the random energy consumption $\tilde{x}_{k, a}^{h}$ in $\mathrm{kWh}$ at each slot $h$ can be described as:

$$
\boldsymbol{I}_{a, k}=\left\{\begin{array}{l}
P_{k, a}^{h+1}=P_{k, a}^{h} M_{k, a}^{h} \\
\tilde{x}_{k, a}^{h}=x_{k, a}^{r a t} M_{k, a}^{h}
\end{array} \quad, M_{k, a}^{h}=\left(\begin{array}{cc}
1-p_{k, a}^{h, f o} & p_{k, a}^{h, f o} \\
p_{k, a}^{h, o f} & 1-p_{k, a}^{h, o f}
\end{array}\right)\right.
$$

where the time varying right stochastic matrix $M_{k, a}^{h}$ is the Markov transition probability matrix and $x_{k, a}^{r a t}$ is the nominal power consumption of the related appliance when is on. Given $M_{k, a}^{h}$ and initial state probability vector $P_{k, a}^{0}$ we can replace the random energy consumption $\tilde{x}_{k, a}^{h}$ with its deterministic value $\hat{x}_{k, a}^{h}$ taking expectation under its probability distribution as follows:

$$
\mathbb{E}\left[\tilde{x}_{k, a}^{h}\right]=\hat{x}_{k, a}^{h}=x_{k, a}^{r a t} P_{k, a}^{0} M_{k, a, h}[0 \quad 1]^{\top}
$$

where $M_{k, a, h}=M_{k, a}^{1} M_{k, a}^{2} \cdots M_{k, a}^{h-1}[24]$.

(II) Semi-inelastic loads: These kinds of appliances have low flexibility for power scheduling. However, the thermostatically on/off operation management of these appliances is very important for ancillary service programs such as frequency regulation. Refrigerators, freezers, and water heaters are three types of thermostatic loads. We show the set of these appliances for consumer $k$ as $\mathcal{A}_{k}^{S I}$. The dynamic equation for such appliances is given by 
$[25,4]:$

$$
T_{k, a}^{h+1}=\epsilon \cdot T_{k, a}^{h}+(1-\epsilon) \cdot\left(T^{0}-\eta \frac{x_{k, a}^{h}}{A}\right), \epsilon=\mathrm{e}^{\left(-\frac{\tau A}{m_{c}}\right)}
$$

where $T_{k, a}^{h}$ is the compartment inner temperature at slot $h, \epsilon$ is the system inertia depending upon the insulation $A$, the thermal mass $m_{c}$ (thermal storage capacity), and the time span $\tau$ between the two time points $h$ and $h+1$. The parameter $x_{k, a}^{h}$ denotes the energy consumption of the appliance at slot $h$ depending on whether the device is on or off, $\eta$ is the efficiency of the device performance and $T^{0}$ describes the ambient temperature, which is assumed to be constant. For this class of appliances the inner temperature is constrained between minimum temperature $T_{k, a}^{\min }$ and maximum temperature $T_{k, a}^{\max }$ as follows:

$$
T_{k, a}^{\min } \leq T_{k, a}^{h} \leq T_{k, a}^{\max }, \forall h \in \mathcal{H}, a \in \mathcal{A}_{k}^{S I}
$$

Further, these appliances must consume a specific energy through the scheduling horizons:

$$
\sum_{h=1}^{H} \Gamma_{k, a}^{h} x_{k, a}^{r a t}=E_{k, a}, a \in \mathcal{A}_{k}^{S I}
$$

where $\Gamma_{k, a}^{h} \in\{0,1\}$ is an integer variable that indicates whether the appliance $a$ of consumer $k$ is in the operating mode (i.e., on) at slot $h$. Moreover, in (5) $E_{k, a}$ is the total electricity demand throughout the scheduling horizon.

(III) Uninterruptible loads: The operation times of these devices are shiftable but are not interruptible and when turned on they should stay on until the completion of their work. Washing machines are such devices. This category is referred to as $\mathcal{A}_{k}^{U N}$. Since the operation of uninterruptible appliances is continuous, we must define the duration of operations $\Delta T_{k, a}$ for these appliances. Moreover, as is clear, each of these appliances have predetermined energy consumption pattern and for each cycle we may have different $x_{k, a}^{r a t}$. So, the task of each consumer is to determine the optimal start time $h^{*}$ for a schedulable appliance $a \in \mathcal{A}_{k}^{U N}$ that minimizes the consumer's payment. This determination can be viewed as selecting one of the cyclic shifts of the total possible power consumption pattern $\chi_{k, a}$ at the scheduling horizon $H$ as follows:

$$
\chi_{k, a}=\left[\begin{array}{cccc}
x_{k, a}^{1} & x_{k, a}^{H} & \cdots & x_{k, a}^{2} \\
x_{k, a}^{2} & \ddots & \cdot & x_{k, a}^{3} \\
\vdots & \cdot & \ddots & \vdots \\
x_{k, a}^{H} & x_{k, a}^{H-1} & \cdots & x_{k, a}^{1}
\end{array}\right], a \in \mathcal{A}_{k}^{U N}
$$

So, to provide the total amount of energy needed for perfect finishing of the operations for each appliance, we should select one of the columns of matrix $\chi_{k, a} \in \mathbb{R}^{H \times H}$ with switch vector $\boldsymbol{\Lambda}_{k, a}$ as:

$$
\boldsymbol{x}_{k, a}=\chi_{k, a} \boldsymbol{\Lambda}_{k, a}
$$

where $\boldsymbol{x}_{k, a} \triangleq\left[x_{k, a}^{1}, \cdots, x_{k, a}^{H}\right]^{\top}$ and $\boldsymbol{\Lambda}_{k, a} \triangleq\left[\Lambda_{k, a}^{1}, \cdots, \Lambda_{k, a}^{H}\right]^{\top}$ are the power consumption profile and the starting time vector of appliance $a$ of consumer $k$ throughout the scheduling horizon $H$ respectively, and $\alpha_{k, a} \leq h^{*} \leq$ $\beta_{k, a}-\Delta T_{k, a}+1$. As the quantities of energy consumption for these appliances are predetermined, we can only determine the start time from its feasible operation window. So, we have:

$$
\sum_{h=\alpha_{k, a}}^{\beta_{k, a}-\Delta T_{k, a}+1} \Lambda_{k, a}^{h}=1, \quad \Lambda_{k, a}^{h} \in\{0,1\}
$$


(IV) Interruptible-continuous loads: These devices are time shiftable and operate in discrete time mode. For these kinds of appliances, the only important thing is that the total energy demand should be provided at the end of their operation window. Including possible interruption devices can be pointed to as plug-in hybrid electric vehicle (PHEV). We show the set of these appliances as $\mathcal{A}_{k}^{I C}$.

$$
\gamma_{k, a}^{\min } \leq x_{k, a}^{h} \leq \gamma_{k, a}^{\max }, \forall h \in \mathcal{H}_{k, a}, a \in \mathcal{A}_{k}^{I C}
$$

where $\gamma_{k, a}^{\min }, \gamma_{k, a}^{\max }$ are minimum standby and maximum power levels which can be consumed by appliance $a$ respectively. To provide the total energy demand for interruptible appliances, we should have:

$$
\sum_{h=\alpha_{k, a}}^{\beta_{k, a}} x_{k, a}^{h}=E_{k, a}, \forall a \in \mathcal{A}_{k}^{I C}
$$

Furthermore, $x_{k, a}^{h}$ in (9) can take all continuous values in this interval and the other constraint applied to this class of appliances is as follow:

$$
x_{k, a}^{h}=0, \forall h \in \mathcal{H} \backslash \mathcal{H}_{k, a}, a \in \mathcal{A}_{k}^{I C}
$$

(V) Interruptible-discrete loads: These appliances are similar to interruptible-continuous loads, except that the energy consumption inside the admissible operation window $\mathcal{H}_{k, a}$ is either off or work at the nominal power rate $\left(x_{k, a}^{h} \in\left\{0, x_{k, a}^{r a t}\right\}\right)$. vacuum cleaner, battery with fixed charge rate, and digital appliances with interruptible jobs are among these devices which is shown as $\mathcal{A}_{k}^{I D}$. Also for these appliances (11) must be satisfied and the total demand constraint is:

$$
\sum_{h=\alpha_{k, a}}^{\beta_{k, a}} \Gamma_{k, a}^{h} x_{k, a}^{r a t}=E_{k, a}, \quad \text { with } \sum_{h=\alpha_{k, a}}^{\beta_{k, a}} \Gamma_{k, a}^{h} \geq 1
$$

(VI) Curtailable loads: In scheduling the curtailable appliances depending on the circumstances, the total energy consumption can take different values. For example, if a consumer has a good financial situation or the price in a given hour is low for him, he can use more power by his curtailable appliances. We show the set of curtailable appliances such as heating, ventilating, and air conditioning (HVAC) systems as $\mathcal{A}_{k}^{C U}$. The dynamic thermal model of curtailable appliances can be represented as follows [8]:

$$
\theta_{a, i n}^{h}\left(\boldsymbol{x}_{k, a}^{h}\right)=\epsilon_{a} \theta_{a, i n}^{h-1}\left(\boldsymbol{x}_{k, a}^{h-1}\right)+\left(1-\epsilon_{a}\right)\left(W_{a, \text { out }}^{h}+K_{a}^{h} x_{k, a}^{h}\right)
$$

where $\theta_{a, i n}^{h}$ is the temperature inside the home at slot $h, \boldsymbol{x}_{k, a}^{h}=\left[x_{k, a}^{1}, x_{k, a}^{2}, \cdots, x_{k, a}^{h}\right], \epsilon_{a}$ is the inertia constant, and $W_{a, \text { out }}^{h}, K_{a}^{h}$ are the outdoor temperature at time slot $h$ and the appliance efficiency, respectively. The consumer $k$ home's temperature is constrained between the minimum acceptable $\left(\theta_{k, a}^{\min }\right)$ and the maximum affordable $\left(\theta_{k, a}^{\max }\right)$ temperatures as follows:

$$
\theta_{k, a}^{\min } \leq \theta_{k, a, i n}^{h} \leq \theta_{k, a}^{\max }, \forall h \in \mathcal{H}, a \in \mathcal{A}_{k}^{C U}
$$

The total energy demand of theses appliances is bounded between the minimum $\left(E_{k, a}^{\min }\right)$ and the maximum $\left(E_{k, a}^{\max }\right)$ energy consumption as follow:

$$
E_{k, a}^{m i n} \leq \sum_{h=\alpha_{k, a}}^{\beta_{k, a}} x_{k, a}^{h} \leq E_{k, a}^{\max }, a \in \mathcal{A}_{k}^{C U}
$$

These bounds can differ for different consumers. 
(VII) Energy Storage loads: The storage devices such as home battery are shown as $a \in \mathcal{A}_{k}^{S}$. The vector scheduled for the energy storage devices at each consumer side is shown as follows:

$$
\boldsymbol{s}_{k} \triangleq \boldsymbol{s}_{k}^{+}+\boldsymbol{s}_{k}^{-} \triangleq\left[s_{k}^{1}, \cdots, s_{k}^{H}\right]
$$

constructed of $s_{k}^{+} \triangleq\left[s_{k}^{+1}, \cdots, s_{k}^{+H}\right]$ and $s_{k}^{-} \triangleq\left[s_{k}^{-1}, \cdots, s_{k}^{-H}\right]$, where $\boldsymbol{s}_{k}^{+}$and $\boldsymbol{s}_{k}^{-}$are vectors of charging and discharging of the storage device, respectively, $s_{k}^{+h} \geq 0$ and $s_{k}^{-h} \leq 0$. $s_{k}^{h}$ is the amount of power scheduled for storage device of consumer $k$ in time slot $h \in \mathcal{H}$. When this consumer stores energy, $s_{k}^{h}$ appears with positive sign and when the storage device is draining with negative sign. Each storage device has limitations for the amount of power that can store or consume according to (17) below. Parameters $B_{k}^{c a p}$ and $B_{k}^{\text {th }}$ indicate maximum and minimum energy levels of the storage device corresponding to consumer $k \in \mathcal{K}$, respectively. $B_{k}^{c a p}$ is the capacity of storage device and $B_{k}^{t h}$ is set to increase the storage device life. The following constraints must be satisfied:

$$
\begin{gathered}
B_{k}^{t h} \leq b_{k}^{h} \leq B_{k}^{c a p}, \forall h \in \mathcal{H} . \\
-\lambda_{k}^{-} b_{k}^{h-1} \leq s_{k}^{-h}, \forall h \in \mathcal{H} . \\
b_{k}^{h}=b_{k}^{h-1}+\lambda_{k}^{+} s_{k}^{+h}-\lambda_{k}^{-} s_{k}^{-h}, \forall h \in \mathcal{H} . \\
s_{k}^{\text {min }} \leq s_{k}^{h} \leq s_{k}^{\text {max }}, \forall h \in \mathcal{H} .
\end{gathered}
$$

where $b_{k}^{h}$ is the storage device charge level for consumer $k$ at slot $h$. We show the storage device charge and discharge efficiency ratio respectively as $\lambda_{k}^{+}$and $\lambda_{k}^{-} \in(0,1]$, which means that a fraction $\lambda_{k}^{+}$of energy consumption of the storage device at slot $h$ is stored as the energy in the storage device and a fraction $\lambda_{k}^{-}$of the stored energy at the previous slot (i.e., $b_{k}^{h-1}$ ) is available for discharge, as denoted in (18). Suppose that the initial energy level of storage device is $b_{k}^{0}$. So, the level of energy stored in slot $h$ follows the dynamic equation (19). The amount of charging and discharging is bounded respectively by $s_{k}^{\max } \geq 0$ and $s_{k}^{\min } \leq 0$, according to (20). For each consumer $k$ we show the set of household devices as vector $\mathcal{A}_{k}=\mathcal{A}_{k}^{I} \cup \mathcal{A}_{k}^{S I} \cup \mathcal{A}_{k}^{U N} \cup \mathcal{A}_{k}^{I C} \cup \mathcal{A}_{k}^{I D} \cup \mathcal{A}_{k}^{C U} \cup \mathcal{A}_{k}^{S}$. The total number of appliances per consumer is shown as $A_{k} \triangleq\left|\mathcal{A}_{k}\right|$.

\subsection{Models for Power System and Appliances}

In the model presented in this paper, $\mathcal{K}$ represents the set of consumers, with its number of consumers as $K \in|\mathcal{K}|$. For every consumer $k \in \mathcal{K}$, the total power demand of retailer at slot $h$ can be shown by $l_{k}^{h}$. In which we have, $h \in \mathcal{H} \triangleq\{1, \cdots, H\}$, where $H$ and $\mathcal{H}$ describe time horizon (e.g. one day ahead) and set of time slots (as integer), respectively. Without loss of generality, for simplicity we assume $H=24$. So, each time slot represents one hour, meaning that $|\mathcal{H}|=24$. The total daily net power demand for each consumer $k$ is denoted by vector $\boldsymbol{l}_{k} \triangleq\left[l_{k}^{1}, \ldots, l_{k}^{H}\right]$. Furthermore, we assume that the retailer has a wind farm and can forecast the power production profile of wind farm $\boldsymbol{r}_{w} \triangleq\left[r_{w}^{1}, \cdots, r_{w}^{H}\right]$ at the beginning of the scheduling horizons according to the explanatory and historical information [26], where $r_{w}^{h}$ is the total power drown from the wind farm at slot $h$. For each appliance $a$, we define an energy consumption scheduling vector $\boldsymbol{x}_{k, a} \triangleq\left[x_{k, a}^{1}, \cdots, x_{k, a}^{H}\right]$, where the scalar $x_{k, a}^{h}$ denotes the corresponding one-hour energy consumption scheduled for appliance $a$ by consumer $k$ at time slot $h$. We further assume that each consumer $k$ similar to retailer, can estimate the internal energy production profile of the PV $\boldsymbol{r}_{k, p} \triangleq\left[r_{k, p}^{1}, \cdots, r_{k, p}^{H}\right]$ 
at the beginning of scheduling horizons, where $r_{k, p}^{h}$ is the total power drowned from the consumer $k$ rooftop PV at slot $h[27]$.

Based on the above definitions the total load demand of the retailer from the $k$ th consumer in time slot $h$ becomes $l_{k}^{h}=\sum_{a \in \mathcal{A}_{k}} x_{k, a}^{h}+s_{k}^{h}-r_{k, p}^{h}$. Each consumer must take the following demand constraint into consideration:

$$
0 \leq l_{k}^{h} \leq l_{k}^{\max }, \forall h \in \mathcal{H}
$$

where $l_{k}^{\max }$ is the maximum amount of power that consumer $k$ can demand from the micro-grid at each slot. Indeed, the constraint (21) ensures that the demand does not exceed the maximum capacity of network. It also prevents the creation of sub-peak at the time slots that the energy price is low. Now, the total power demand by all consumers throughout the scheduling horizon $H$ is defined by $\boldsymbol{l} \triangleq\left[l^{1}, \cdots, l^{H}\right]$ with aggregate demand at slot $h$ as:

$$
l^{h}=\sum_{k \in \mathcal{K}} l_{k}^{h}-r_{w}^{h}=\sum_{k \in \mathcal{K}}\left(\sum_{a \in \mathcal{A}_{k}} E_{k, a}+\boldsymbol{s}_{k}-\boldsymbol{r}_{k, p}\right)-r_{w}^{h}
$$

\subsection{Dynamic Pricing and PAR}

One of the most important parameters in measuring the usefulness of any DR program is ability to reduce the peak to average energy consumption ratio (i.e., PAR) as much as possible which has several positive impacts on the power system [28]. The PAR level throughout one scheduling horizon $H$ is calculated as:

$$
\mathrm{PAR}=\frac{l_{\text {peak }}}{l_{\text {avg }}}, l_{\text {peak }}=\max _{h \in \mathcal{H}} l^{h}, l_{\text {avg }}=\frac{1}{H} \cdot \sum_{h \in \mathcal{H}} l^{h}
$$

The simulation results show that by increasing the number of consumers equipped with storage devices to a certain number, the PAR decreases. But after that, the PAR will start to increase again. This is because those users who have storage devices tend to buy more energy before the peak hours and consume or sell it during the peak hours to maximize their profit. If a large number of consumers do this, the original peak energy consumption moves to a new peak in another hour. We call this phenomenon reverse peak or sub peak. This destructive effect can be resolved using dynamic pricing methods such as real time pricing (RTP) method provided in this paper. Let $\mathcal{P}=\left[\mathcal{P}^{1}, \cdots, \mathcal{P}^{H}\right] \in \mathbb{R}^{H}$ be a set of real time prices (RTP) for each determined time interval by the retailer in which, $\mathcal{P}^{h}$ is the price at slot $h$ that the retailer updates and send it to the consumers. Let $\overline{\boldsymbol{V}}_{\boldsymbol{k}}=\left[\boldsymbol{v}_{k, 1}, \cdots, \boldsymbol{v}_{k, A_{k}}\right] \in \mathbb{R}^{H_{n e w} \times A_{k}}$ be the set of virtual time prices (VTPs) determined by the consumer $k$ 's smart meter when any change occurs in the consumer $k$ state, where $H_{n e w}=H-t_{k}+1$ and $t_{k}$ is the time slot when a change occurs for consumer $k . \boldsymbol{v}_{k, a}=\left[v_{k, a}^{1}, \cdots, v_{k, a}^{H_{n e w}}\right]^{\top}$, where $\top$ refers to vector transpose, is just the virtual price vector that the consumer $k$ 's smart meter broadcasts to schedule the appliance $a$ energy consumption when a change occurs. Actually, VTP is a tool to transmit and manage the change command, control the power usage, and prioritize the appliances.

It should be noted that, if after scheduling, for whatever reason, a change is introduced to the price trends (the retailer side), all the consumers must adapt their load profiles according to the new state, but if the change occurs in a consumer condition, preferences, or objective function, only that consumer (using VTP) must adapt his load profile accordingly. In this situation the main challenge to make any changes is that, the last state of the uninterruptible appliances should be checked again and if they are turned on, they should stay on until finishing their 
Table 1: Summary of Notations.

\begin{tabular}{|c|c|c|c|}
\hline Symbol & Description & Symbol & Description \\
\hline $\mathcal{H}_{k, a}, \mathcal{H}_{n e w}$ & $\begin{array}{l}\text { Set of permissible time slots for scheduling operation of appliance } a \text { of } \\
\text { consumer } k \text { and the updated scheduling horizon within the day }\end{array}$ & $T_{k, a}^{h}, T_{k, a}^{\min }$ & $\begin{array}{l}\text { The compartment inner temperature of appliance } a \text { of customer } k \text { at slot } h \\
\text { and the minimum inner temperature bound }\end{array}$ \\
\hline$\alpha_{k, a}, \beta_{k, a}$ & $\begin{array}{l}\text { Minimum start time of operation for appliance } a \text { of consumer } k \text { and the } \\
\text { maximum end time of operation }\end{array}$ & $T_{k, a}^{\max }$ & Maximum inner temperature bounds of appliance $a$ of consumer $k$ \\
\hline $\mathcal{H}, H, h, t_{k}$ & $\begin{array}{l}\text { Set of equal length time slots, scheduling horizon, time slot index, and the time } \\
\text { slot when a change occurs for consumer } k\end{array}$ & $\theta_{a, i n}^{h}, \theta_{k, a}^{\min }$ & $\begin{array}{l}\text { The inside temperature at home at slot } h \text { regarding appliance } a \text { and the } \\
\text { minimum inner temperature bound }\end{array}$ \\
\hline $\mathcal{K}, K, k$ & Set of consumers, total number of consumers, and their index & $\theta_{k, a}^{\max }$ & Maximum inner temperature bounds of appliance $a$ of consumer $k$ \\
\hline $\mathcal{A}_{k}^{I}, \mathcal{A}_{k}^{S I}$ & Set of consumer $k$ 's inelastic and semi-inelastic loads & $\chi_{k, a}, \Lambda_{k, a}$ & $\begin{array}{l}\text { Total possible power consumption pattern of appliance } a \text { of consumer } k \text { and } \\
\text { the switch vector }\end{array}$ \\
\hline $\mathcal{A}_{k}^{U N}, \mathcal{A}_{k}^{I C}$ & Set of consumer $k$ 's uninterruptible and interruptible-continuous loads & $P, P^{h}$ & The day-ahead vector of real time prices (RTP) and the price at slot $h$ \\
\hline $\mathcal{A}_{k}^{I D}, \mathcal{A}_{k}^{C U}$ & Set of consumer $k$ 's interruptible-discrete and curtailable loads & $P^{\min }, P^{\max }$ & $\begin{array}{l}\text { Minimum and maximum prices that the retailer can } \\
\text { offer at each hour }\end{array}$ \\
\hline $\mathcal{A}_{k}^{S}, s_{k}^{+}, s_{k}^{-}$ & $\begin{array}{l}\text { Set of consumer } k \text { 's energy storage loads, vectors of charging and } \\
\text { discharging }\end{array}$ & $C_{h^{(}}^{(\cdot), U_{k, a}(\cdot)}$ & $\begin{array}{l}\text { The energy cost at the wholesale market and the utility function of appliance } \\
a \text { of consumer } k\end{array}$ \\
\hline$E_{k, a}^{\min }$ & Minimum tolerable energy demand bounds of appliance $a$ of consumer $k$ & $\bar{V}_{k} v_{k, a} v_{k, a}^{h}$ & $\begin{array}{l}\text { Virtual time price (VTPs) matrix for consumer } k \text {, the VTP vector } \\
\text { corresponding to appliance, and the VTP at slot } h\end{array}$ \\
\hline$E_{k, a}^{\max }$ & Maximum tolerable energy demand bounds of appliance $a$ of customer $k$ & $\stackrel{h}{w} w_{k, a}, m_{k, a}$ & $\begin{array}{l}\text { Priority of energy consumption index at slot } h \text { related to appliance a of } \\
\text { consumer } k \text { and the median level of energy demand }\end{array}$ \\
\hline$x_{k, a}, x_{k, a}^{h}$ & $\begin{array}{l}\text { Daily energy consumption vector of appliance } a \text { and the corresponding one- } \\
\text { hour energy consumption scheduled for the appliance }\end{array}$ & $U_{k}^{\min }, c^{\max }$ & $\begin{array}{l}\text { Minimum satisfaction level for consumer } k \text { and the maximum amount of } \\
\text { costs imposed on the consumers bound }\end{array}$ \\
\hline$r_{w}, r_{w}^{h}$ & Power production profile of wind farm and the corresponding one-hour output & $\mathcal{A}_{k}, A_{k}, a$ & $\begin{array}{l}\text { Set of customer } k \text { 's appliances, total number of these appliances, and } \\
\text { appliance index }\end{array}$ \\
\hline$r_{k, p}, r_{k, p}^{h}$ & $\begin{array}{l}\text { The internal energy production profile of consumer } k \text { 's PV and the } \\
\text { corresponding one-hour output }\end{array}$ & $l_{k}, l_{-k}, l_{k}^{h}$ & $\begin{array}{l}\text { Daily net power demand for consumer } k \text {, net demand for the other } \\
\text { consumers, and the total energy demand of the consumer at time slot } h\end{array}$ \\
\hline$E_{k, a}, x_{k, a}^{r a t}$ & $\begin{array}{l}\text { Total desirable energy need of appliance } a \text { of consumer } k \text { for finishing its task } \\
\text { and the nominal power consumption of the appliance }\end{array}$ & $l^{h}, l_{k}^{\max }$ & $\begin{array}{l}\text { The aggregate purchased power from the market at slot } h \text { and the maximum } \\
\text { amount of power that consumer } k \text { can demand }\end{array}$ \\
\hline$\gamma_{k, a}^{\min }, s_{k}^{\min }$ & Minimum power level that appliance $a$ of consumer $k$ can consume & $y, Y, x, X$ & $\begin{array}{l}\text { integer variable index, set of feasible regions for these variables, continuous } \\
\text { variable index, set of feasible regions for these variables }\end{array}$ \\
\hline$\gamma_{k, a}^{\max } s_{k}^{\max }$ & Maximum power level that appliance $a$ of consumer $k$ can consume & $d, \rho^{i}$ & $\begin{array}{l}\text { An acceptable step of the trust-region SQP method with component } d_{x}, d_{y} \text {, } \\
\text { and the trust-region radius }\end{array}$ \\
\hline$B_{k}^{t h}, B_{k}^{c a p}$ & $\begin{array}{l}\text { Minimum and maximum energy levels of the storage device corresponding to } \\
\text { consumer } k\end{array}$ & $\theta, p$ & The integrality gap and the experimental order of convergence \\
\hline$b_{k}^{h}, \lambda_{k}^{+}, \lambda_{k}^{-}$ & $\begin{array}{l}\text { The storage device charge level for consumer } k \text { at slot } h \text { with charge and } \\
\text { discharge efficiency ratio }\end{array}$ & $Q_{k}(\bullet), W^{(i)}$ & $\begin{array}{l}\text { Function which expresses the personal restrictions for each consumer for the } \\
\text { adaptive scheduling stage and approximation of the Hessian of the Lagrangian }\end{array}$ \\
\hline
\end{tabular}

operations. To do this, the states of these appliances must be set as the initial conditions for the new optimization model [6]. The main notations ans synbols of the proposed framework are listed in Table 1.

\section{Problem Formulation}

\subsection{Retailer Price Control as Leader (Stage 1)}

From the retailer perspective, the strictly convex function $C_{h}\left(l^{h}\right)$ represents the cost of providing electricity from the wholesale market at each hour $h$. By defining the minimum and maximum prices that the retailer can offer at each hour as $\mathcal{P}^{\text {min }}$ and $\mathcal{P}^{\text {max }}$, we have:

$$
\mathcal{P}^{\text {min }} \leq \mathcal{P}^{h} \leq \mathcal{P}^{\text {max }}, \forall h \in \mathcal{H}
$$

$\mathcal{P}^{\text {min }}$ and $\mathcal{P}^{\max }$ are usually designed based on many factors such as price history, market competition, customers' acceptability and the wholesale price. The retailer must bear in mind that if he wants to compete in the electricity market and has a positive effect on the consumption patterns, there is an upper bound for determining the price rate/signal for it. So, $\mathcal{P}^{\max }$ can be marked as this upper bound. Because of external (such as political) pressure and consumers' acceptability, the retailer must limit his scheduling methodology in a way that, the maximum amount of costs imposed on the consumers does not exceed the limit $c^{\max }$. As a result, we have the following constraint:

$$
\sum_{h \in \mathcal{H}} \mathcal{P}^{h} \cdot\left(l^{h}+r_{w}^{h}\right) \leq c^{\max } .
$$


However, there is a cost imposed on the retailer for providing the power, $C_{h}\left(l^{h}\right)=\nu^{h} \cdot\left(l^{h}\right)^{2}+b^{h} \cdot\left(l^{h}\right)+c^{h}$, representing the energy cost that can be either a real energy cost (as a result of using the fossil fuels, buying power from the wholesale market), or a managed virtual cost for encouraging the consumers to participate in the optimization program [17]. Here, $\nu^{h}>0$ and $b^{h}, c^{h} \geq 0$ are referred to as the price parameters determined at the wholesale market for time slot $h \in \mathcal{H}$ and $l^{h}$ is the total consumed energy at that slot. Finally, using (22) the profit maximization problem can be modeled as:

$$
\max _{\mathcal{P}^{h}} \sum_{h \in \mathcal{H}}\left(\mathcal{P}^{h} \cdot \sum_{k \in \mathcal{K}}\left(\sum_{a \in \mathcal{A}_{k}} x_{k, a}^{h}+\boldsymbol{s}_{k}-\boldsymbol{r}_{k, p}\right)-C_{h}\left(l^{h}\right)\right) \text { s.t. }(24),(25)
$$

Problem (26) shows that the cost imposed on the consumers due to the optimization variable $\mathcal{P}^{h}$ is directly affected by their aggregate consumption $\sum_{k \in \mathcal{K}} l_{k}^{h}$ and the wholesale price parameters $\left\{\nu^{h}, b^{h}, c^{h}\right\}$. As the objective function (26) and constraint (24) are linear and constraint (25) is nonlinear, this non-linear optimization problem can be solved efficiently using a non-linear solver such as interior point method (IPM) [29].

\subsection{Consumer Price Control as Follower (Stage 1)}

We assume that each consumer wishes to minimize his payment subject to a satisfaction level more than a certain threshold. So, all the consumers try to reduce their electric bills as much as possible subject to an acceptable satisfaction level. Therefore, each consumer first determines his desired utility (satisfaction), then, seeks to find a schedule which minimizes his bill. This constraint is presented as:

$$
\sum_{a=1}^{A_{k}} U_{k, a}\left(x_{k, a}^{h}, \omega_{k, a}^{h}\right) \geq U_{k}^{\min }
$$

where $U_{k}\left(x_{k, a}^{h}, \omega_{k, a}^{h}\right)=\sum_{h=1}^{H} 1-e^{\omega_{k, a}^{h}\left(1-\left(x_{k, a}^{h} / m_{k, a}\right)\right)}$ is the concave utility function describing the quality of energy consumption of appliance $a$ of consumer $k$ throughout the scheduling horizons [30], and $m_{k, a} \geq 0$ is the median level of energy demand of this appliance [10]. The appliance $a$ prioritization is performed through $\omega_{k, a}^{h}$. A higher $\omega_{k, a}^{h}$ at slot $h$ implies higher priority for energy consumption at this slot. For example, let us assume that the consumer $k$ wants to use his washing machine at slot $h$ (i.e, $\omega_{k, a}^{h}$ has the highest value for this slot and it reduces monotonically as $h$ approaches $H$ ). Without the DR program, this consumers' satisfaction level (utility) regarding the washing machine is $100 \%$, as he operates this appliance right at slot $h$. Now, let us assume that the price signal at this slot is high and the ECS decides to operate the washing machine at slot $h+n$ (i..e, $n$ slots delay in the operation time). This delay incurs some dissatisfaction to the consumers which is modeled by $U_{k}\left(x_{k, a}^{h}, \omega_{k, a}^{h}\right)$. So, the ECS should make a trade-off between delaying the operation time to reduce the payment and reducing the time delay $n$ to increase the satisfaction level. The optimization problem at each consumer side becomes:

$$
\begin{aligned}
& \max _{x_{k}}-\left(\sum_{h=1}^{H} \mathcal{P}^{h} \cdot\left(l_{k}^{h}+l_{-k}^{h}\right)\right)=\min _{x_{k}} \sum_{h=1}^{H} \mathcal{P}^{h} \cdot\left(l_{k}^{h}+l_{-k}^{h}\right), \\
& \text { s.t. }(2)-(5),(7)-(15),(17)-(21),(27)
\end{aligned}
$$

where $l_{-k}^{h}$ is aggregated demand of all the consumers other than consumer $k$ at slot $h$ and we denote the vector of aggregate demand for all $h \in \mathcal{H}$ by $\boldsymbol{l}_{-k}$. Without constraints (21) and (27) the problem can be decomposed into a number of sub-problems each corresponding to an appliance and each sub-problem can be solved relatively 
easily [8]. However, in our problem the variables for appliances are coupled due to constraints (21) and (27). Besides, the integer variables regarding appliances $a \in \mathcal{A}_{k}^{S I} \cup \mathcal{A}_{k}^{U N} \cup \mathcal{A}_{k}^{I D}$ make the problem even more complex and challenging. In order to solve this problem, we use an efficient method inspired by the integrated filter based Sequential Quadratic Programming (SQP) and Branch-and-bound (B\&B) method [31].

\subsection{The Globalized SQP Algorithm}

The problem introduced in Eq. (28) is an MINLP (because of having nonlinear constraints and both integer and the availability of continuous variables), which is hard to solve. One way to solve an MINLP problem is to decompose it into non-linear and integer parts. Due to high computational complexity, these methods are not often cost-effective. To address this issue, in our distributed framework we use an integrated trust-region approach [31] for solving the MINLP. This method uses a B\&B technique ${ }^{1}$, but at the consumer side, the non-linear part (NLP) of problem is not optimally solved. Instead, branching is allowed after each iteration of the NLP solver. In this way, the non-linear (continuous) part of the MINLP problem is solved whilst searching the tree (integer part).

To solve an MINLP with B\&B, initially, all the integer restrictions are relaxed and the resulting NLP relaxation is solved. If the obtained solution of the relaxed NLP problem assigns integer values to all integer variables, then this solution also solves the MINLP. Usually, some integer variables take a non-integer value. The algorithm then selects one of those integer variables which takes a non-integer value, say $y^{(i)}$, with value $\hat{y}^{(i)}$, and branches on it. The branching operation generates two new NLP problems by adding simple bounds $y^{(i)} \leq\left[\hat{y}^{(i)}\right]$ and $y^{(i)} \geq\left[\hat{y}^{(i)}\right]+1$ respectively to the NLP relaxation (where $[z]$ is the largest integer not greater than $z$ ). One of the two new NLP problems is selected and solved next. If the integer variables take non-integer values then branching is repeated, thus generating a branch-and-bound tree whose nodes correspond to NLP problems and where an edge indicates the addition of a branching bound. If one of the following fathoming rules is satisfied, then no branching is required, the corresponding node has been fully explored (fathomed) and can be abandoned. The fathoming rules are; FT1an infeasible node is detected (in this case the whole sub-tree starting at this node is infeasible and the node has been fathomed), FT2- an integer feasible node is detected (this provides an upper bound on the optimum of the MINLP; no branching is possible and the node has been fathomed), FT3- a lower bound on the NLP solution of a node is greater than or equal to the current upper bound (in this case the node is fathomed, since this NLP solution provides a lower bound for all problems in the corresponding sub-tree). Once a node has been fathomed the algorithm backtracks to another node and terminates when all the nodes are fathomed [32]. The non-linear solver considered in this method is a SQP solver ${ }^{2}$ and the global convergence is promoted through the use of a trust-region with the new concept of a filter [33]. Fortunately, the quadratic programs are easy to solve in the sense that there are good procedures for their solution.

\footnotetext{
${ }^{1} \mathrm{~A} B \& \mathrm{~B}$ algorithm searches the complete space of solutions for a given problem for the best solution. However, explicit enumeration is normally impossible due to the exponentially increasing number of potential solutions. The use of bounds for the function to be optimized combined with the value of the current best solution enables the algorithm to search parts of the solution space only implicitly. B\&B has three main components: selection of the node (unexplored subspaces) to process, bound calculation, and branching (i.e., subdivision of the solution space of the node into two or more subspaces to be investigated).

${ }^{2} \mathrm{SQP}$ is an iterative method for obtaining the solution to non-linear constrained optimization problems. As with most optimization methods, SQP is not a single algorithm, but rather a conceptual method from which numerous specific algorithms have evolved.
} 
The general trust region method minimizes an unconstrained objective by imposing an artificial constraint on the step length. Typically, this constraint says that the step length is less than some length, or $\|d\|<\Delta$, where $d$ is the step length, $\Delta$ is the maximum step distance (often considered as the "radius" of the trust region), and $\|\cdot\|$ is some norm defined in $\mathbb{R}^{n}[34]$. The size of the trust-region decreases if the step is refused and increases if it is accepted. Because of space limitation, we briefly introduce the formulation by only considering the nonlinear constraint (27). However, the other linear constraints can be applied in similar way. For each consumer, we define $\mathbf{f}(x, y)$ and $\mathbf{g}(x, y)$ as:

$$
\begin{aligned}
\mathbf{f}(x, y) & =\sum_{h=1}^{H} \mathcal{P}^{h} \cdot l_{k}^{h} \\
\mathbf{g}(x, y) & =U_{k}^{m i n}-\sum_{a=1}^{A_{k}} U_{k, a}\left(x_{k, a}^{h}, \omega_{k, a}^{h}\right) .
\end{aligned}
$$

where variables $x$ and $y$ are constructed of all continuous and discrete variables in $l_{k}^{h}, x_{k, a}^{h}$, and $\omega_{k, a}^{h}$, respectively. Consider an NLP problem at a given node of the branch-and-bound tree, we now formulate the NLP part of problem in $(28)$ as

$$
\begin{aligned}
& (P)=\left\{\begin{array}{l}
\min _{x, y} \mathbf{f}(x, y), \\
\text { s.t. } \mathbf{g}(x, y) \leq 0, x \in X, y \in Y \text { integer. }
\end{array}\right. \\
& (\hat{P})=\left\{\begin{array}{l}
\min _{x, y} \mathbf{f}(x, y) \\
\text { s.t. } \mathbf{g}(x, y) \leq 0, x \in X, y \in \hat{Y} \text { integer. }
\end{array}\right.
\end{aligned}
$$

where, as mentioned, each $y$ is an integer variable belonging to the decision set $(y \in\{0,1\})$, from $a \in \mathcal{A}_{k}^{S I} \cup \mathcal{A}_{k}^{U N} \cup \mathcal{A}_{k}^{I D}$ and $x$ are continuous variables belonging to $a \in \mathcal{A}_{k}^{I C} \cup \mathcal{A}_{k}^{C U} \cup \mathcal{A}_{k}^{S} . X$ and $Y$ are sets of a feasible region for this appliance, respectively. Note that, $\hat{Y} \subset Y$ involves further constraints added during branching. In this case the integer restrictions are relaxed and since both $\mathbf{f}(x, y)$ and $\mathbf{g}(x, y)$ are convex, we have a convex MILP [29]. Finally, we apply the integrated MINLP approach to our problem $(\hat{P})$, which results in solving a sequence of quadratic programming $(\mathrm{QP})$ problems of the form

$$
\left(Q P_{c, \infty}^{i}\right)=\left\{\begin{array}{l}
\min _{d}\left(\mathbf{f}^{(i)}+\nabla_{x, y} \mathbf{f}^{(i)^{T}} d+1 / 2 d^{T} W^{(i)} d\right) \\
\text { s.t. } \mathbf{g}^{(i)}+\nabla_{x, y} \mathbf{g}^{(i)^{T}} d \leq 0, \\
\mathbf{f}^{(i)}+\nabla_{x, y} \mathbf{f}^{(i)^{T}} d \leq U-\epsilon, \\
\|d\|_{\infty} \leq \rho^{i}, \\
x^{(i)}+d_{x} \in X, y^{(i)}+d_{y} \in \hat{Y} \text { integer. }
\end{array}\right.
$$

where $\mathbf{f}^{(i)}=\mathbf{f}\left(x^{(i)}, y^{(i)}\right)$ and $\mathbf{g}^{(i)}=\mathbf{g}\left(x^{(i)}, y^{(i)}\right)$ at iteration $(i)$. Further, $d$ is an acceptable step of the trust-region SQP method with components $d_{x}$ and $d_{y}$ in accordance with the variables $x$ and $y$, respectively. For approximation of the Hessian of the Lagrangian we use $W^{(i)} \approx \nabla^{2} L^{(i)}=\nabla_{x, y}^{2} \mathbf{f}^{(i)}+\sum \lambda_{n} \nabla_{x, y}^{2} \mathbf{g}_{n}^{(i)}$. Also, the parameter $\lambda_{n}$ is the Lagrange multiplier for $\mathbf{g}_{n}(x, y) \leq 0$. The third term of (33) refers to a mechanism for terminating the progression of $\left(Q P_{c, \infty}^{i}\right)$ when an underestimator (e.g. $\left.(\hat{\mathbf{f}})\right)$ exceeds the upper bound $U$ of the branch-and-bound process. At 
the same term $\epsilon>0$ is an acceptable tolerance for the branch-and-bound. The trust-region radius $\rho^{i}$ in the fourth term is adjusted to ensure that the quadratic model $\left(Q P_{c, \infty}^{i}\right)$ complies with the true function. The SQP method solves the NLP using a sequence of QP approximations which is achieved by replacing the non-linear constraints with a linear first order Taylor series approximation and replacing the non-linear objective by a second order Taylor series approximation reinforced by second order information as constraints. Although under certain conditions the SQP method converges quadratically to a solution, the use of trust-region may cause $\left(Q P_{c, \infty}^{i}\right)$ to be infeasible even though the problem without a trust-region has a non-empty feasible region. So, a feasibility restoration phase is necessary to get closer to the feasible region (to ensure convergence to a feasible solution) by minimizing the violation of the constraints in some norm, subject to the linear constraints $x \in X, y \in \hat{Y}$ as follow:

$$
(F)=\left\{\begin{array}{l}
\min _{x, y}\left\|h^{+}(x, y)\right\|, \\
\text { s.t. } x \in X, y \in \hat{Y} \text { integer. }
\end{array}\right.
$$

where $h(x, y)=\left(\begin{array}{c}\mathbf{f}(x, y)-(U-\epsilon) \\ \mathbf{g}(x, y)\end{array}\right)$ The operation $a^{+}=\max (0, a)$ is performed component wise. Hence, a SQP algorithm also minimizes $(F)$. We have shown all these procedures in Algorithm 1. In this algorithm, round $(y)$ is the nearest integer to $y$. Fathoming of nodes occurs when the lower bound exceeds the current upper bound, when the sub-problem is infeasible or when all integer variables $y_{n}$ take on discrete values.

Theorem 1. Since $\mathbf{f}(x, y)$ and $\mathbf{g}(x, y)$ are smooth and convex functions, $X$ and $Y$ are convex sets, the number of integer variables $y$ is finite, and the underlying trust-region SQP method is globally convergent, Algorithm 1 converges to the unique optimal solution $\left(x^{*}, y^{*}\right)$ of $(P)$ after visiting a finite number of nodes.

Proof. See Appendix A.

In step (I) of Algorithm 1 in order to efficiently integrate the restoration phase using the trust-region we must compute an acceptable step $d^{(i)}$ to make sure of feasibility. In (II) we have proposed a fathoming approach (i.e., FT1, FT2, and FT3) of Lemma 1 to remove the infeasible solutions. The main idea of branch-and-bound method is mentioned in steps (III) and (IV). In implementing the proposed technique, it is likely that some integer variables converge to a non-integral solution, namely $y_{n}^{(i)} \rightarrow \hat{y}_{n}$ but $\theta \simeq 0.02<\tau$, i.e. the integrality gap remains bounded away from zero (we can let $\tau=0.1[35]$ ), or the SQP solver converges at second order rate during this time and $\theta \gg\left\|d^{(i)}\right\|_{\infty} \rightarrow 0$. So, during the early branching procedure we have to first compute the integrality gap in step (V) and the experimental order of convergence in step (VI). Then, in step (VII) we add the constraint to take advantage of the quadratic rate of convergence and prevent inactivity in the early branching process during the second order convergence of the SQP method ${ }^{3}$.

\subsection{Virtual Electricity-Trading Process (Stage 2)}

Many DR studies introduce a day-ahead pricing framework, which sometimes has significance difference from the applied RTP $[36,17,37,5,6]$. The reason for this difference is imprecision in the estimation and unanticipated

\footnotetext{
${ }^{3}$ Interested readers can view more information of this scheme and its features in [31].
} 


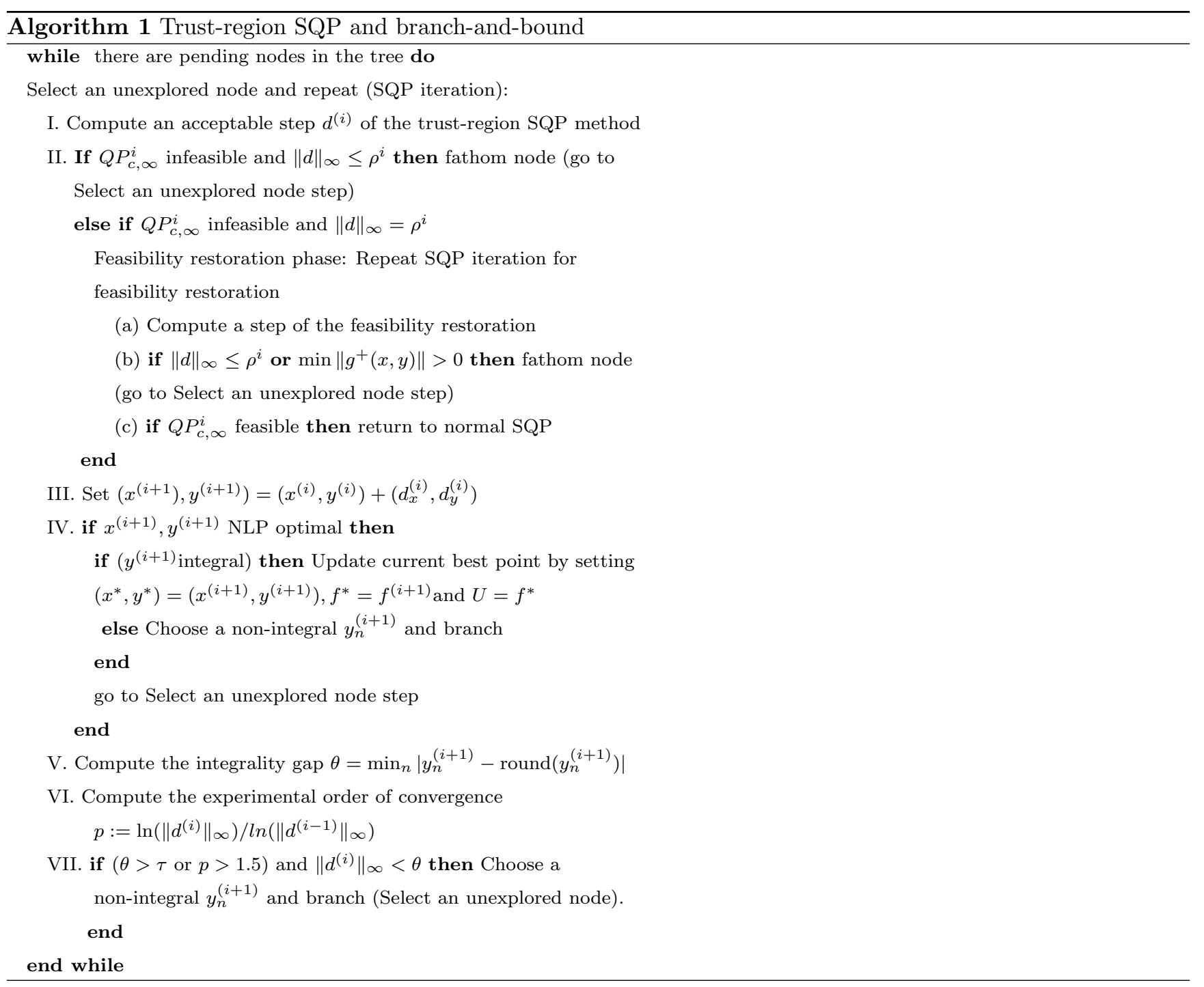


events. In this work we want to reduce the consequences of this difference by means of adaptive rescheduling energy consumption in respond to the occurred change which is based on event-triggered model predictive control methods [38]. In this way, we propose a complementary figurative RTP-based electricity-trading Stackelberg strategy for determination of optimal load rescheduling of appliances when an event (change) is occurred at the consumer side. We use this algorithm to run a figurative electricity-trading Stackelberg process, in which the consumer's smart meter is the leader who imposes virtual time prices (VTP), and its appliances are as followers.

Remark 1. This framework does not mean that each appliance must work independently and in a distributed manner. Indeed, in this stage, all the calculations for making optimal decisions can be done centrally by the ECS, either. However, the difference between this framework and convex disciplined optimization methods is that this is a more general method as the optimization method can be viewed as a special case of game theory, i.e. it involves mixed and behavioral strategies as well as pure strategies. In the proposed method the ECS seeks to achieve an equilibrium in a cooperative manner (which is more flexible target for our framework) instead of minimum/maximum point on equal strategies, with the following advantages: 1- Each appliance as an active decision maker can be controlled centrally by ECS or directly by itself with different strategies in different situations which improves the practicality and the consumer satisfaction level. 2- The consequence of decision by each appliance directly impacts on the other appliances. So, all the appliances can adapt to the new condition in real time when a change is occurred.

Device Model: Each appliance as an ECS followers, selects an optimal reaction from its strategy set for minimizing its incommodity function. Let $\overline{\boldsymbol{V}}_{k}=\left[\boldsymbol{v}_{k, 1}^{\top}, \cdots, \boldsymbol{v}_{k, A_{k}}^{\top}\right]$ be the strategy of this consumer's ESC, where each element $\boldsymbol{v}_{k, a}=\left[v_{k, a}^{1}, \cdots, v_{k, a}^{H}\right]$ corresponds to the VTP vector determined for device $a \in \mathcal{A}_{k}$ and $A_{k}=\left|\mathcal{A}_{k}\right|$ is the number of appliances for consumer $k$. When $\overline{\boldsymbol{V}}_{k}$ is revealed to device $a$, given the designated VTP for this appliance, it has the following incommodity function:

$$
\varphi_{k, a}\left(\overline{\boldsymbol{V}}_{k}, \boldsymbol{x}_{k, a}, \boldsymbol{\omega}_{k, a}\right)=\boldsymbol{v}_{k, a} \boldsymbol{x}_{k, a}^{\top}-w_{k, a} \cdot U_{k, a}\left(\boldsymbol{x}_{k, a}, \boldsymbol{\omega}_{k, a}\right)
$$

where, $\boldsymbol{v}_{k, a} \boldsymbol{x}_{k, a}^{\top}$ is the cost resulting from the demand energy $\boldsymbol{x}_{k, a}, U_{k, a}\left(\boldsymbol{x}_{k, a}, \boldsymbol{\omega}_{k, a}\right)$ is the utility resulting from the consuming energy (see (27)), and $w_{k, a} \geq 0$ is a weight factor that reveals the significance of the corresponding appliance energy consumption. As device $a$ aims to minimize the incommodity, the optimization problem for each device is formulated as:

$$
\min _{x_{k, a}} \varphi_{k, a}\left(\overline{\boldsymbol{V}}_{k}, \boldsymbol{x}_{k, a}, \boldsymbol{\omega}_{k, a}\right) \quad \text { s.t. } \quad x_{k, a}^{\min } \leq x_{k, a}^{h} \leq x_{k, a}^{\max }
$$

where, $x_{k, a}^{\min }$ and $x_{k, a}^{\max }$, are minimum and maximum energy consumption levels determined by consumer $k$ for appliance $a$ in slot $h$ when the program changes. We assume that this problem is strictly convex, therefore, has unique solution [39, 29].

ECS Model: The figurative profit obtainable by the ECS through this scheme is modeled as:

$$
U_{k, E C S}\left(\overline{\boldsymbol{V}}_{k}, \overline{\boldsymbol{X}}_{k}\right)=\sum_{a=1}^{A_{k}} \boldsymbol{v}_{k, a} \boldsymbol{x}_{k, a}^{\top}-\sum_{a=1}^{A_{k}} \boldsymbol{\mathcal { P }} \boldsymbol{x}_{k, a}^{\top}
$$

Here, $\boldsymbol{v}_{k, a} \boldsymbol{x}_{k, a}^{\top}$ and $\mathcal{P} \boldsymbol{x}_{k, a}^{\top}$ denote the profit and cost resulted from appliance $a$, respectively. In this sense, the ECS determines $\boldsymbol{v}_{k, a}$ for each appliance based on the strategy profile $\overline{\boldsymbol{X}}_{k}=\left[\boldsymbol{x}_{k, 1}^{\top}, \cdots, \boldsymbol{x}_{k, A_{k}}^{\top}\right]$ coming back from the 
appliances. Afterward, each appliance receives the vector $\boldsymbol{v}_{k, a}$ and update its strategy $\boldsymbol{x}_{k, a}$ and send it to the ECS. This procedure continues until convergence. The profit maximization for the ECS is proposed as:

$$
\max U_{k, E C S}\left(\overline{\boldsymbol{V}}_{k}, \overline{\boldsymbol{X}}_{k}\right) \text { s.t. } Q_{k}\left(\overline{\boldsymbol{V}}_{k}, \overline{\boldsymbol{X}}_{k}\right)
$$

where $Q_{k}($.$) is a function which expresses the personal restrictions for each consumer. This function can be different$ for different consumers and is a private information.

\section{Event-triggered Stackelberg Game}

\subsection{Existence and Uniqueness of Stackelberg Equilibrium}

Consider Stackelberg game $\mathscr{G} \triangleq\{\mathscr{P}, \mathscr{S}, \mathscr{U}\}$, where $\mathscr{P}$ is the set of players (all the consumers as well as the retailer), $\mathscr{S} \triangleq\left\{\mathscr{S}_{j}\right\}_{j \in \mathscr{P}^{4}}$ is an $(K+1)$-tuple of the pure strategy sets (total energy demand vector satisfying set of personal constraints for each player), and $\mathscr{U} \triangleq\left\{\mathscr{U}_{j}\right\}_{j \in \mathscr{P}}$ is an $(K+1)$-tuple of the payoff functions (objective function of each player).

Definition 1. A strategy profile $\boldsymbol{S}^{*} \in \mathscr{S}$ constitutes the Stackelberg equilibrium (SE) of game $\mathscr{G}$, if and only if it satisfies the following set of inequalities.

$$
\begin{gathered}
\mathscr{U}_{k}\left(S_{k}^{*}, \boldsymbol{S}_{-k}^{*}\right) \geq \mathscr{U}_{k}\left(S_{k}, \boldsymbol{S}_{-k}^{*}\right), \forall S_{k} \in \mathscr{S}_{k}, k \in \mathcal{K} \\
\mathscr{U}_{R}\left(S_{R}^{*}, \boldsymbol{S}_{-R}^{*}\right) \geq \mathscr{U}_{R}\left(S_{R}, \boldsymbol{S}_{-R}^{*}\right), \forall S_{R} \in \mathscr{S}_{R}
\end{gathered}
$$

where $S_{k}^{*}, \boldsymbol{S}_{-k}^{*}, S_{R}^{*}$, and $\boldsymbol{S}_{-R}^{*}$ are the optimal strategies of consumer $k$, the optimal strategies of all the players other than consumer $k$, the optimal strategies of the retailer, and the optimal strategies of all the players except the retailer, respectively.

Let's consider the mentioned game as a bi-level optimization problem consists of retailer maximization problem (26) subject to consumers minimization problem (28) as follows [40]:

$$
\begin{array}{r}
\max _{S_{R} \in \mathscr{S}_{R}} U_{R}\left(S_{R}, \boldsymbol{S}_{-R}\right) \\
\text { s.t., } S_{k} \in \underset{S_{k} \in \mathscr{S}_{k}}{\operatorname{argmax}} U_{k}\left(S_{k}, S_{R}\right), \forall k \in \mathcal{K}
\end{array}
$$

Theorem 2. The bi-level optimization problem (41) has unique optimal solution and this solution is SE of game $\mathscr{G}$ for stage 1.

Proof. See Appendix B.

At stage 2, it is clear that the demand of each consumer's appliances (the reaction strategy) is subject to the total available capacity, and also depends on the occurred event. So, for this stage we term the formulated game $\mathscr{G}$ as generalized Stackelberg game (GSG). Since the followers' strategies are coupled, they need to seek a generalized Nash equilibrium (GNE) instead of a traditional Nash equilibrium. In particular, we are interested in investigating

\footnotetext{
${ }^{4} j \in\{k$ (consumer index $), R($ retailer index $\left.)\right\}$
} 
the existence and properties of a variational equilibrium ${ }^{5}(\mathrm{VE})$, which is a type of GNE. This is due to the fact that a VE is more socially stable than any other GNE (if there exists any) and thus, it is a desirable network state [41].

Theorem 3. For a fixed virtual price vector $\overline{\boldsymbol{V}}_{\boldsymbol{k}}$, a socially optimal VE exists in the proposed game $\mathscr{G}$, between consumer $k \in \mathcal{K}$ as the leader and his appliances as the followers.

Proof. See Appendix C.

\subsection{Solution Methods for the Proposed Games}

In this section we propose two different algorithms for the scenarios in Section 3. The Stackelberg game can be interpreted as a sequential game played between one leader and some followers [42]. So, in our adaptive game, in stage 1 the retailer plays the role of leader and determines the RTP, while the users are followers and schedule their load profiles to only minimize the cost functions. In this stage the satisfaction function is not considered explicitly (due to the privacy security consideration ${ }^{6}$ ) and there is only a lower bound $U_{k}^{\min }$ for the total satisfaction level. Then in stage 2, each user becomes an autonomous virtual leader and its appliances play the role of virtual followers and seek to maximize the satisfaction level. To follow the changes in the consumer state during the scheduling horizon, the figurative electricity-trading process is triggered adaptively to reschedule the operation of the appliances in response to the update in the consumer preferences or renewed production. This improves the energy bill reduction, users satisfaction, and preserving operational constraints within limits. The whole procedure is summarized and depicted in Fig. 2. From preceding section result, the charge for each consumer depends on how he and all other users schedule their consumptions. This naturally leads to the game process among Retailer and consumers proposed in Algorithm 2 and game process among smart meter's ECS and its appliances in Algorithm 3. In these schemes, the proposed one leader N-follower Stackelberg game model consists of sequential decision-making problem and its traditional proposed solution, is the sub-game perfect equilibrium (SPE). To formulate the SPE we can use Backward Induction as a common approach, which starts from the last action and reasons backwards [43]. At stage 1, we use IPM algorithms to obtain the solution for the retailer and integrated SQP for solving consumer's objective functions, but at stage 2, because of change in the objective functions we use normal SQP to optimize the ECS and the objective functions of its appliances [34]. Obviously, this theorem is strategy-proof and the users do not benefit from misleading each other by providing inaccurate information about their usage during their interactions [10]. Finally, we introduce two constraints for determination of the initial values in Algorithm 3 as:

$$
\begin{gathered}
\mathcal{P}^{\text {old }}=\mathcal{P}^{0}, \mathcal{P}^{\text {new }}=\mathcal{P} \forall h \in \mathcal{H}_{\text {change }} \\
\boldsymbol{x}_{k, a}^{\text {old }}=\boldsymbol{x}_{k, a}^{0}, \boldsymbol{x}_{k, a}^{\text {new }}=\boldsymbol{x}_{k, a} \forall h \in \mathcal{H}_{k, \text { change }}, a \in \mathcal{A}_{k}
\end{gathered}
$$

\footnotetext{
${ }^{5}$ The variational inequality problem, $\operatorname{VI}(\mathbf{X}, \mathbf{F}(x))$, consists in finding a vector $\bar{x} \in \mathbf{X}$ such that $(y-\bar{x})^{\top} \cdot \mathbf{F}(\bar{x}) \geq 0 \forall y \in \mathbf{X}$.

${ }^{6}$ For taking into account utility function $U_{k}\left(x_{k, a}^{h}, \omega_{k, a}^{h}\right)$ in the optimization problem (28) as an objective function, each consumer must share parameters such as $x_{k, a}^{h}, \omega_{k, a}^{h}$, and $m_{k, a}$ to make sure about the convergence of the corresponding game. However, such parameters are the private information of each consumer and most of the consumers are not willing to share it neither with the retailer nor with the other consumers.
} 


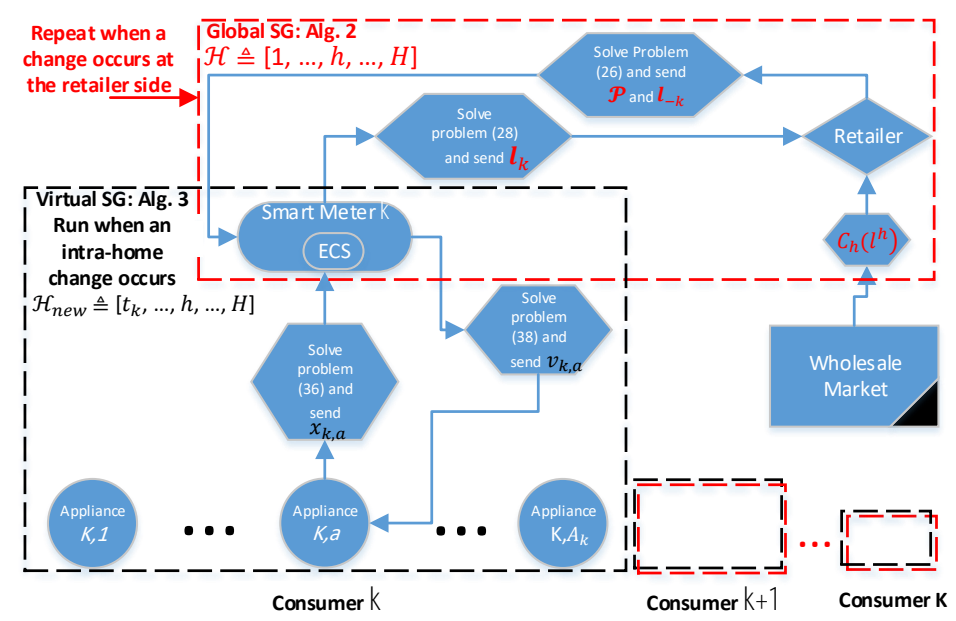

Figure 2: Block diagram of the proposed adaptive event-triggered game-based DR.

where, $\mathcal{P}^{\text {new }} \triangleq\left[\mathcal{P}^{t}, \cdots, \mathcal{P}^{H}\right]$ and $\boldsymbol{x}_{k, a}^{\text {new }} \triangleq\left[x_{k, a}^{t_{k}}, \cdots, x_{k, a}^{H_{k}}\right]$ emerge as a result of change in system and $\mathcal{P}^{0}, \boldsymbol{x}_{k, a}^{0}$ are initial price vector and load profile of appliance $a$ of consumer $k$ respectively. $\mathcal{H}_{\text {change }}=\{t, \cdots, H\}$ and $\mathcal{H}_{k, \text { change }}=\left\{t_{k}, \cdots, H_{k}\right\}$ are the set of remaining time slots from when the change is occurred for retailer $(t)$ or for consumer $k\left(t_{k}\right)$ to the end of scheduling horizon, respectively ${ }^{7}$.

\section{Simulation Results}

\subsection{Simulation Setup}

In our considered benchmark smart micro-grid system, we have $K=10$ consumers. For the purpose of performance comparison, each user is selected to have 2 appliances with inelastic operation such as TV, PC or lighting system. We divided the set of these appliances as background and digital appliances. We assumed that each consumer has 2 appliances with semi-elastic operation such as refrigerator or freezer. Moreover, each consumer is also assumed to have 2 appliances with uninterruptible operation, i.e., dishwasher, washing machine or clothes dryer, 2 appliances with interruptible continuous operation such as PHEV or pool pump, 2 appliances with interruptible discrete operation such as vacuum cleaner or computers with interruptible jobs, and 2 appliances with curtailable operation such as air conditioner or ventilator.

We assume that each consumer can determine the capacity of the compressor of his semi-elastic appliances, Markov transition probabilities of inelastic loads, and the energy consumption bound of his curtailable appliance according to the historical data and characteristics of his appliances. So, to satisfy constraint (14), based on dynamic equation (13), (insulating) properties of the building, and experimental data we can bound the consumption level of curtailable appliances as $\underline{u}_{k, a}^{h} \leq x_{k, a}^{h} \leq \bar{u}_{k, a}^{h}$ at each slot $h$. To satisfy constraint (4) we assume that the compressors of semi-elastic appliances need two time slots until the temperature of the appliance change from $T_{k, a}^{\min }$ to $T_{k, a}^{\max }$. Moreover, we assumed that each consumer has one rooftop PV and the retailer has one wind farm, but the capacity of each PV is assumed to be a small fraction of the daily requirement of each consumer, so that everyone has the

\footnotetext{
${ }^{7}$ All these modifications can be done in a similar way for Algorithm 2 when a change is occurred in the retailer side.
} 


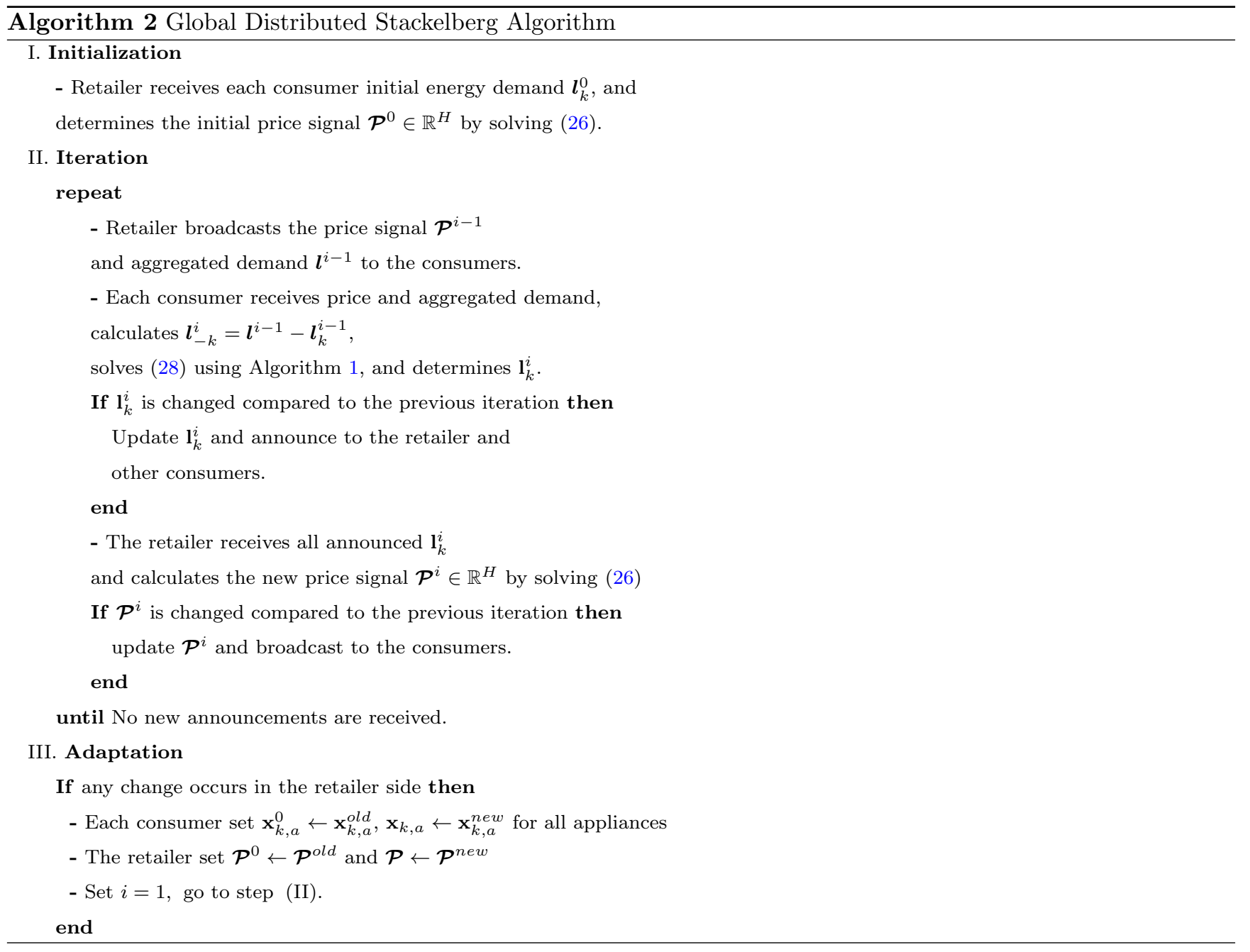




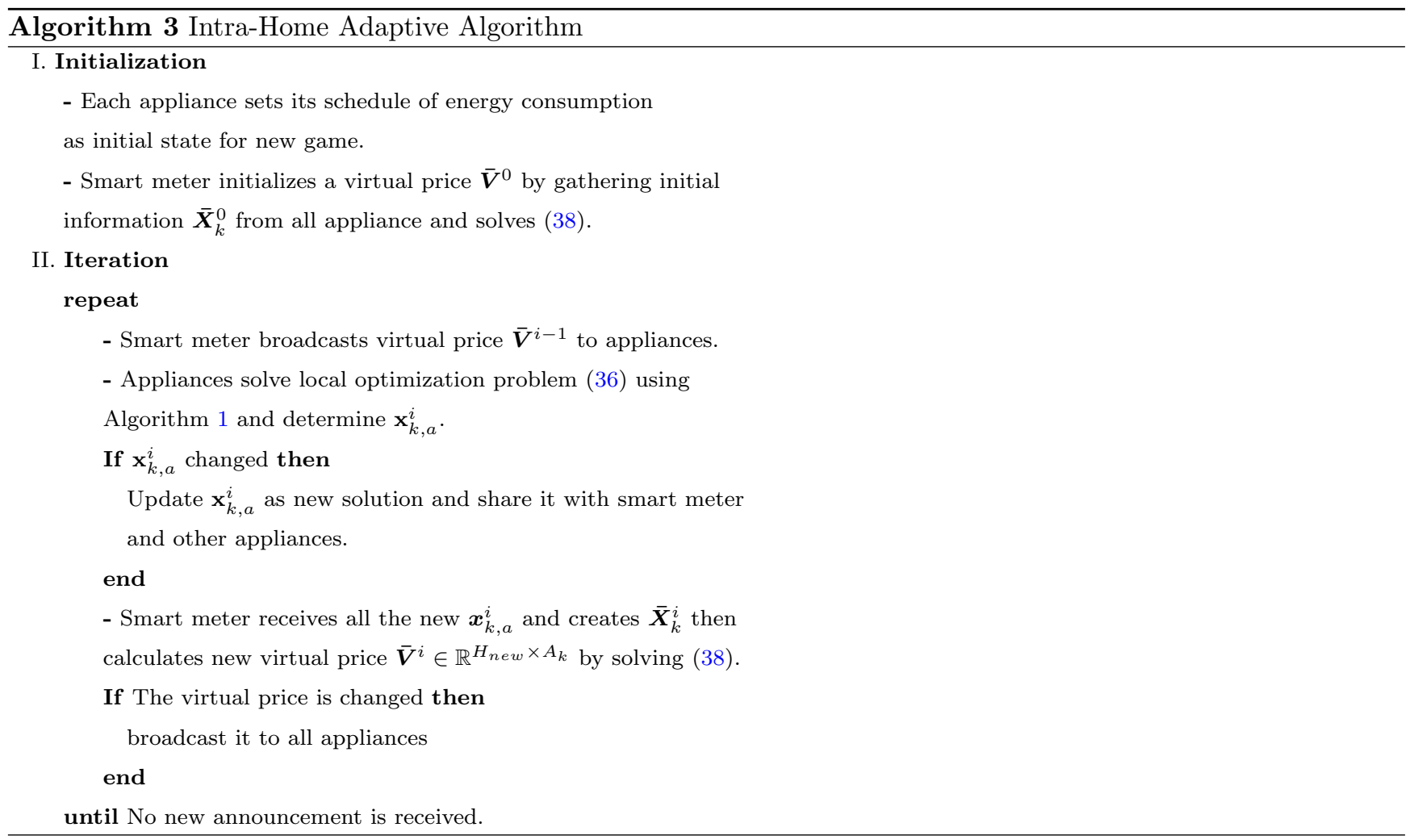

incentive to participate in the game. We listed the set of these appliances and their individual requirements in Table 2. In addition, for simplicity, without loss of generality we assumed that for the quadratic cost function, $C_{h}\left(l^{h}\right)$ referred to in Section 3.1, we have $b^{h}, c^{h}=0, \boldsymbol{\nu}^{8}=(0.15,0.12,0.1,0.1,0.2,0.3,0.45,0.45,0.5,0.6,0.6,0.6,0.5,0.4$, $0.45,0.5,0.6,0.8,0.9,1,1.1,0.9,0.7,0.5)\left(\right.$ cents $\left./ k W h^{2}\right)$, for battery $\lambda_{k}^{+}, \lambda_{k}^{-}=1$, there is not any charge/discharge limit and the efficiency of PHEV is $100 \%$. Note that, for curtailable appliances the utility is defined based on the amount of energy consumption per slot, but for uninterruptible appliances the utility reduces based on the distance from the starting time window $\left(\alpha_{k, a}\right)$ and for other appliances we let $\omega_{k, a}^{h}=0$.

\subsection{The Attractiveness to Retailer and Power Company}

At this part, we want to evaluate our proposed event-triggered DR method by comparing it with the case in which a constant pricing plan has been adopted. Accordingly, in the constant pricing plan (without the DR program) the price signal is independent of consumers' behavior. We assumed that in this scheme the consumers use their appliances at their nominal power and whenever need to turn on an appliance, they immediately use it. For example, they use their appliances precisely at the start time of scheduling window $\mathcal{H}_{a}$. Further, we let $5 \times \nu^{h} \leq \mathcal{P}^{h} \leq 15$ at each hour $h$. For a better comparison, in the optimal constant pricing model we used the same parameters as our model parameters. In Fig. 3 we have drawn the operation of retailer and consumers in both cases. Indeed, in this figure, we have plotted total power profile of consumers to compare the PAR and the price signal determined by the retailer before and after applying our method. Note that, PAR is one of the most important factors for network stability measure, so the retailer and power company tries to reduce this parameter.

${ }^{8}$ Here $\nu$ is the vector of all the price parameters coming from the wholesale market through one day, i.e., $\nu \triangleq\left[\nu^{1}, \cdots, \nu^{H}\right]$ 
Table 2: Parameters for residential appliances

\begin{tabular}{l|c|c|c|c|c|c|c|c}
\hline Appliance name & $\begin{array}{c}E_{k, a}^{\min } \\
(\mathrm{kWh})\end{array}$ & $\begin{array}{c}E_{k, a}^{\max } \\
(\mathrm{kWh})\end{array}$ & $\begin{array}{c}\gamma_{k, a}^{\min } \\
(\mathrm{kW})\end{array}$ & $\begin{array}{c}\gamma_{k, a}^{\max } \\
(\mathrm{kW})\end{array}$ & $\begin{array}{c}\underline{u}_{k, a}^{\min } \\
(\mathrm{kWh})\end{array}$ & $\begin{array}{c}\bar{u}_{k, a}^{\max } \\
(\mathrm{kWh})\end{array}$ & $\alpha_{k, a}$ & $\beta_{k, a}$ \\
\hline Background appliances & 2.5 & 2.5 & 0.1 & 0.1 & - & - & $1: 00$ & $24: 00$ \\
Digital appliances & 1.56 & 1.56 & 0.13 & 0.13 & - & - & $1: 00$ & $24: 00$ \\
Refrigerator & 1.32 & 1.32 & 0 & 0.11 & - & - & $1: 00$ & $24: 00$ \\
Freezer & 1.2 & 1.2 & 0 & 0.1 & - & - & $1: 00$ & $24: 00$ \\
Washing machine & 1.94 & 1.94 & 0 & $0.97-0.97$ & - & - & $9: 00$ & $21: 00$ \\
Dish washer & 1.44 & 1.44 & 0 & $1-0.44$ & - & - & $20: 00$ & $7: 00$ \\
Vacuum cleaner & 2.2 & 2.2 & 0 & 0.55 & - & - & $15: 00$ & $24: 00$ \\
Computers & 1.2 & 1.2 & 0 & 0.2 & - & - & $15: 00$ & $10: 00$ \\
PHEV & 9.9 & 9.9 & 0 & 1.98 & - & - & $18: 00$ & $8: 00$ \\
Pool pump & 12 & 12 & 0 & 3 & - & - & $14: 00$ & $8: 00$ \\
Air conditioner & 7.2 & 12 & 0 & 0.7 & 0.12 & 0.5 & $1: 00$ & $24: 00$ \\
Ventilator & 3.6 & 6 & 0 & 0.4 & 0.05 & 0.25 & $1: 00$ & $24: 00$ \\
Battery & 0 & 0 & -2.1 & 2.1 & - & - & $1: 00$ & $24: 00$ \\
\hline
\end{tabular}

Our simulation shows that the PAR in optimal constant pricing becomes 2.4241 while in our scheme it is 1.4595 , which shows $39.7924 \%$ improvement. In Fig. 3 we see that in the case of constant pricing there is high price rate at the peak demand which makes the consumers to shift a lot of their demand to off peak demand. This behavior creates sub-peaks at slots with low price. By contrast, in our schemes, the price is changed dynamically with change in the total power consumption which prevents creation of sub-peaks.

In this work, we assume that all the consumers have battery with similar specifications and we see that in our proposed dynamic pricing, the battery performance does not create sub-peaks in the demand profile, whereas if we use constant pricing, when the number of consumers equipped with storage devices goes beyond almost half, the PAR goes out of the optimal state. This is because a large number of consumers shift their consumption too much, leading to an increase in PAR (creating sub-peaks) [37]. Note that, without use of batteries in the proposed DR, PAR becomes 1.5617 which has 7\% increase compared with that of our approach. Furthermore, the revenue for the retailer before DR is $\$-10.76$ and after DR is $\$ 84.11$, the average amount of constant price before DR is about 13.7 (cents/kWh) and average amount of real-time price with DR is 12.6 (cents/kWh). This means without the DR, there is not much economic attraction for the retailer.

On the other hand, the trends of resulting total PAR and energy cost during the distributed iterations of our method are shown in Fig. 4. We can see that the proposed distributed algorithm converges quickly and as the 


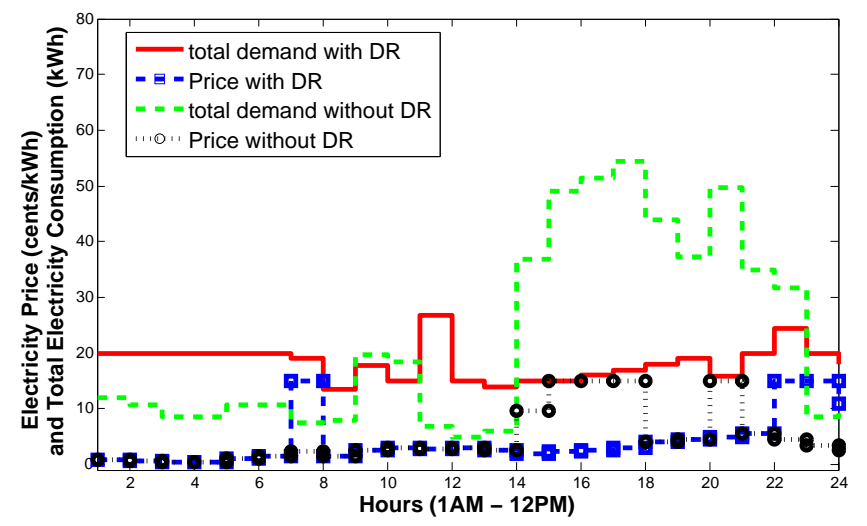

Figure 3: Benefit for the retailer before and after the DR program.
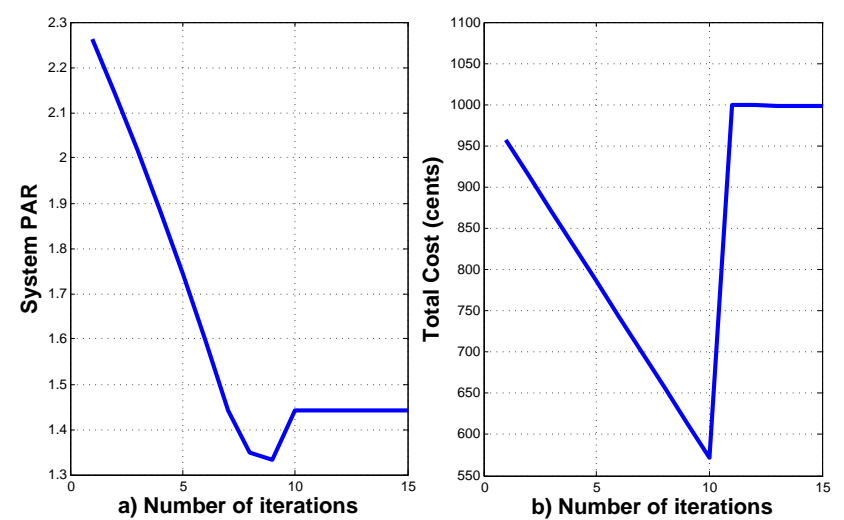

Figure 4: Convergence of the resulting PAR and the energy cost using the proposed ASG: (a) convergence of PAR; (b) convergence of cost.

users run the algorithm, it converges after about 10 iterations only, i.e., around 1 iteration per user on average. Moreover, the simulations show that the proposed adaptive pricing approach significantly increases the retailer profit, whilst reduces the total consumer expenses up to $44.17 \%$, in comparison with optimal constant pricing. The peculiar behavior in the convergence of Fig. 4 (b) returns to Stackelberg theory. According to this theory, the retailer determines the price signal at first and then the consumers try to minimize their payments by scheduling their energy consumption patterns. So, we can expect that the total cost reduces monotonically as each consumer updates his energy consumption schedule until the last consumer operation (iteration 10). After that, according to the consumer's behavior the retailer updates his price signal (at iteration 11) in order to maximize his benefit. So, we can expect that the total cost curve has sudden increase. After the retailer action the algorithm is converged and no one has incentive to deviate from the resulted equilibrium.

For another comparison and showing the computational performance of the presented scheme the effect of increasing the number of customers (i.e., the size of the power system) on the total computation time in the network is depicted in Fig. 5. In this figure the scenario "SG-B\&B" is the case at which the system participants' interactions are modeled by the Stackelberg game while the optimization solution is based on the classical branchand-bound method. In the "VSG-B\&B" scenario, the participants' interactions strategy is based on the VCG design presented in [17]. Finally, the case at which all the consumers' data are gathered at the retailers side and the DR 


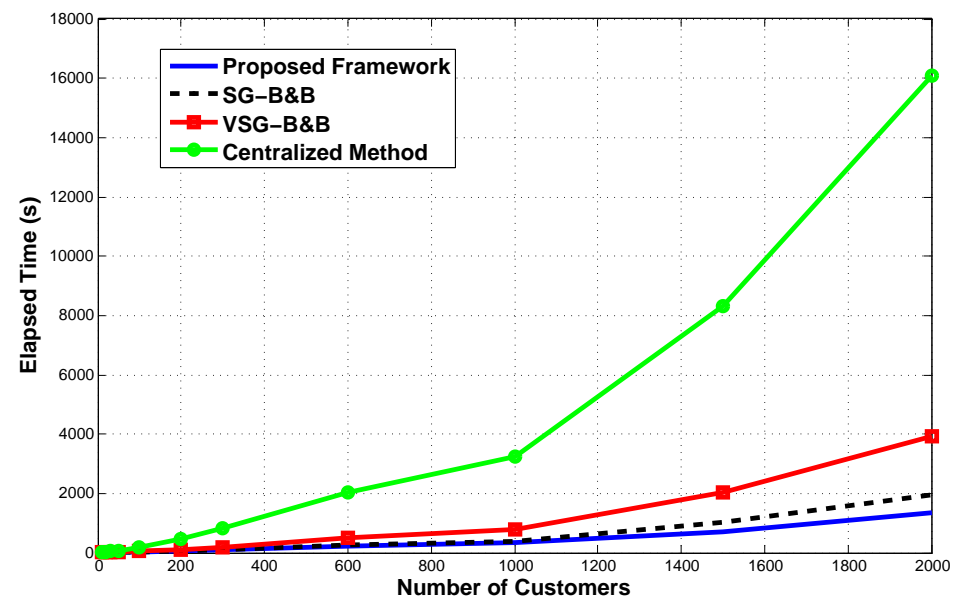

Figure 5: Comparisons between the proposed framework and other scenarios, in terms of computation time.

problem is solved centrally by the retailer is denoted by scenario "Centralized Method". As denoted, our framework imposes the lowest computational burden on the system. The first reason is that using Stackelberg game, the DR program converges to an optimal solution after only 2 iterations per consumer (as we saw in Fig. 4). This is why our framework imposes lower communication and computational overhead compared to mechanisms like "VSG-B\&B". The other reason is using the integrated trust-region approach in our framework to deal with the integer variables, which is shown in [31] to be more effective compared to the classical B\&B technique like "SG-B\&B". As is clear in Fig. 5, the centralized solutions are not practical in real-world real-time applications as the required time to achieve an optimal solution is way too long. It is worth mentioning that the results under different simulation setups (e.g., communication delay, the total number of appliances, percentage of appliances $\mathcal{A}_{k}^{S I}, \mathcal{A}_{k}^{U N}, \mathcal{A}_{k}^{S D}$, etc.) and the PC laptop hardware can change and lead to more significant differences. For example, increasing the percentage of appliances like $\mathcal{A}_{k}^{S I}, \mathcal{A}_{k}^{U N}, \mathcal{A}_{k}^{S D}$, increases the number of integer variables in the DR problem, which in turn increases the elapsed time to converge for all the scenarios and the gap between our framework and the other solutions.

\subsection{The Attractiveness to Consumers}

In this part, we analyze the benefits gained by the consumers from participating in the DR program. The simulation results of the total cost in which each consumer must pay for two scenarios is denoted in Fig. 6. For without DR program case we assumed that each consumer uses his appliances right whenever he needs and there is not any cooperation with the retailer and other consumers for intelligent and conscious use of energy. Further, for the simulation scenario, we also assumed that each consumer randomly has between 2 to 6 appliances of each class defined in Section 2.1. So, the total number of appliances for each consumer varies between 12 and 36 . As is clear from Fig. 6, by using our proposed approach, the total payment for each consumer is much less than when there is not any DR program.

For analyzing stage 2 of the proposed ASG scheme, we show the effectiveness of the method when a change occurs in the consumer side. To design this change, we assume that the change occurred at time 12:00 AM for consumer 3 and because of the high sun's radiation, the power production of rooftop PV of this consumer is increased by 


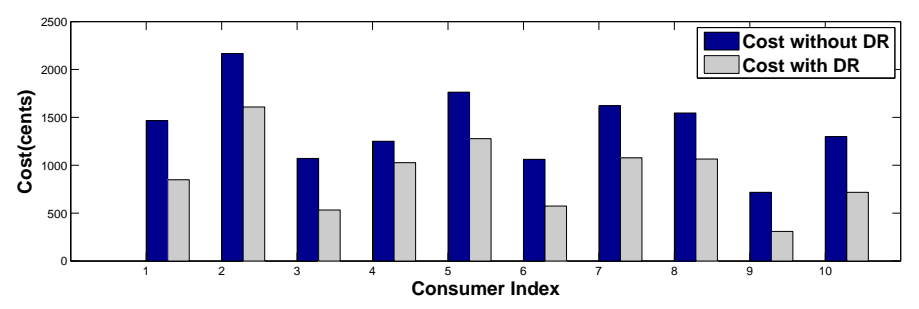

Figure 6: Total cost imposed on the consumers with/without the proposed framework.

$1.3 \mathrm{kWh}$. We assume that in this case the consumers only wish to increase the use of curtailable appliances with increasing the priority factor $\left(\omega_{k, a}\right)$ for this type of appliances. So, this consumer does not change the scheduling for appliances $\mathcal{A}_{k}^{I}, \mathcal{A}_{k}^{S I}$ and $\mathcal{A}_{k}^{U N}$. We have depicted the simulation results in Fig. 7 . The results show that after adopting our adaptive scheme, the level of consumer welfare increases by $1 \%$. However, this increase may be less noticeable, whereas in larger scales with more appliances and more changes this gain can be very significant. Further, the level of welfare strictly depends on the consumer priority factors $\omega_{k, a} a \in \mathcal{A}_{k}$ and can change among different consumers and different situations. As you can see in Fig. 7, in addition to the battery, to improve the welfare and adapting to the new conditions, the battery inside the PHEV is also used to supply power. We can see that, from total excess power production, AC has consumed $1.1 \mathrm{kw}$ and $0.2 \mathrm{kw}$ has been used for ventilation, because $\mathrm{AC}$ is allocated more priority factor compared to ventilation. Moreover, the negative power consumption of pool pump means that the appliance can change its energy consumption pattern and consume less energy in those slots. However, the total power consumption of all appliances throughout the scheduling horizons is not reduced in this scheme and the increased power consumption of curtailable appliances is equally to increase in the PV power production as in Fig. 8. This figure shows that the total revenue of the retailer and total PAR are not changed in the adaptive stage of our framework and there is no concern about them or also about ancillary service problems.

In another simulations, the total cost imposed on the consumers in different situations is listed as in Table 3. According to these results we can find that this framework is useful even for consumers who do not participate in the program (denoted as DR-Disobey). But we must note that as the total number of consumers who do not participate in the proposed game increased, the performance deteriorates (i.e., the retailer revenue's reduces and there will be imbalance in supply and demand).

To further denote the adaptive stage performance of our framework we considered another scenario in which the power line between the consumer 5 and the retailer is interrupted and the blackout is occurred. As depicted in Fig. 9, after the blackout, without the DR program all appliances of consumer 5 are off. However, with our event-triggered scheme after the blackout the energy stored in the battery and PHEV is used to feed the critical appliances such as lighting and heating/cooling systems. In other words, using priority factor $\omega_{k, a}$, the consumer can prioritize his appliances and keep going on with those which are most necessary.

To evaluate the optimality of the proposed DR method, we have compared the performance of our framework with three scenarios under a real-time pricing protocol. For the simulation setup we have randomly assigned different appliances to different consumers and used real-time price signal data from 5/10/2019 to 5/11/2019 for pricing node ID 3 (zone MID-ATL/APS) of Pennsylvania-New Jersey-Maryland Interconnection (PJM) electricity market [44]. 


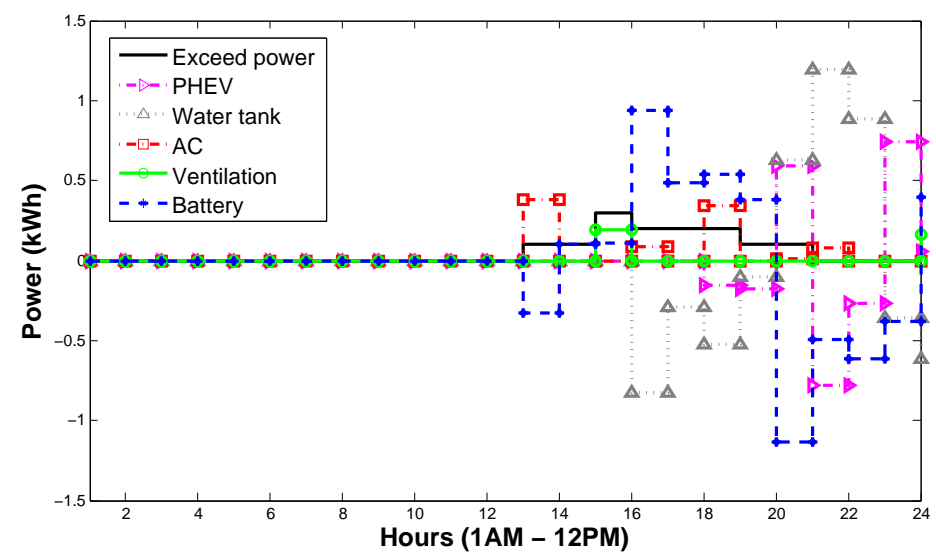

Figure 7: Performance of appliances when power production of PV increased.

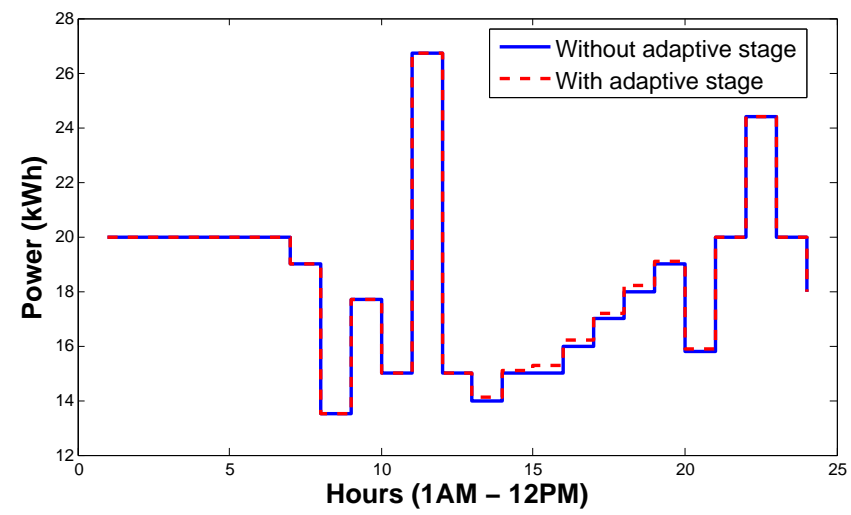

Figure 8: Total power consumption with/without adaptive stage.

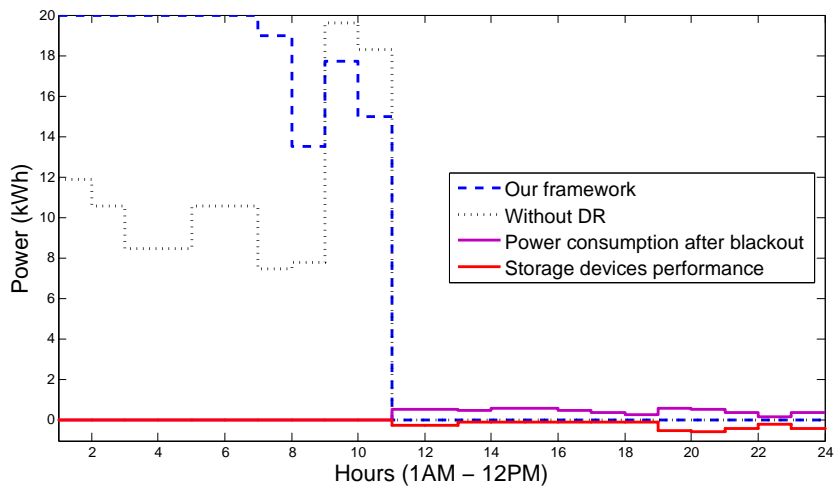

Figure 9: Performance of our approach for consumer 5 when the blackout occurred at slot 10 .

Table 3: Comparison of total cost in micro-grid

\begin{tabular}{c|c|c|c|c}
\hline States & Without DR & DR & DR-Disobey & DR without battery \\
\hline $\begin{array}{c}\text { Total cost } \\
\text { (Dollars) }\end{array}$ & 161.21 & 90 & 104.17 & 91.24 \\
\hline \hline
\end{tabular}




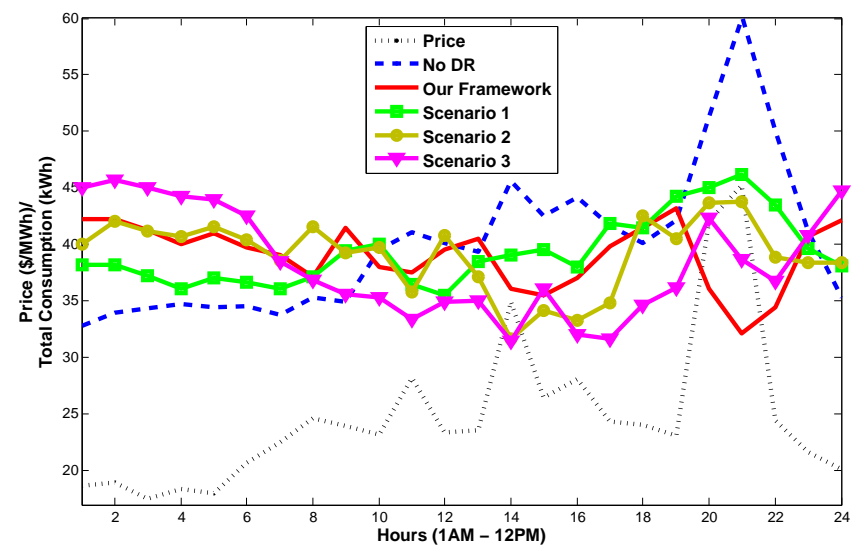

Figure 10: Performance comparison of different DR mechanisms under real-time pricing protocol.

For the first DR scenario (i.e., scenario 1 in Fig. 10), we implemented the Stackelberg game solution provided in [10]. As depicted in Fig. 10, the aggregate power consumption in our framework and scenario 1 are much more flat than the case No-DR. Total PAR for No-DR case is 2.9682, while for our framework and scenario 1 they are 1.6186 and 1.6457, respectively. However, as in our framework all the home devices are modeled in details and the solution is adaptive, the DR program is much more flexible. From the figure, the high flexibility in our solution makes it possible for the consumers to significantly decrease their consumption rate when the price signal has a sudden increase. For scenario 1 it is not possible for the consumers to effectively reduce their consumptions when the price has the highest level (e.g., time slots 21 and 22) due to the low flexibility in modeling the home appliances and renewable sources. The cost imposed on the consumers in our framework is $\$ 87.34$ while for scenario 1 the aggregate cost is $\$ 96.42$. For the second scenario (i.e., scenario 2 in Fig. 10), we applied the solution mechanism provided in [16] to the DR problem.

As depicted, in scenario 2, the consumers are not able to consume the least amount of power when the price rate is the highest. The first reason is due to lack of detailed modeling of residential devices which results in low flexibility in the energy consumption scheduling. The second reason is the lack of adaptivity to price changes which leads to inaccurate evaluation of the price value. The aggregate PAR in this case is 1.6026 and the total cost is $\$ 93.61$. For scenario 3, we used the design model in [17]. Under this DR solution, the consumers are not able to adapt to the changes in the real-time price and power production values. Besides, similar to the other scenarios, this solution has also low flexibility because of not considering the detailed model for the residential devices. That is why the aggregate consumption curve doesn't reach to the lowest level when the price signal has the highest amount. For this scenario the aggregate PAR is 1.6687 while the aggregate cost is $\$ 92.84$.

\section{Conclusion}

In this paper, we developed a multi-objective distributed optimization framework in which by controlling the multi-class appliances the PAR, the consumer's bill, and the incommodity level are minimized and the retailer profit maximized. To achieve this, we proposed a novel event-triggered game-theoretical two stage decision-making scheme for the electricity retailer and his consumers. We modeled multiple appliances in six categories and used an 
incommodity function to characterize their welfare levels. The interaction between consumers with their appliances and the retailer was modeled as an adaptive real-time pricing Stackelberg game. In addition, we verified that such schemes can deal with unforeseen events and increase the quality of power usage and ancillary services. Moreover, for solving the MINLP program using our model, unlike conventional methods that are based on decomposition, we used an integrated SQP and B\&B algorithm which is faster, more accurate and requires less computation cost. The ideas in this work can be extended in several directions. For example, a smart grid with multiple retailers can be considered and an analysis can be performed when the consumers are also able to sell power to the main grid.

\section{Appendix A. Proof of Theorem 1}

Lemma 1. Let $\mathbf{f}(x, y)$ and $\mathbf{g}(x, y)$ be smooth and convex functions. A sufficient condition for fathoming rule $\boldsymbol{F R} 3$ applied to $(\hat{P})$ to be satisfied is that any $Q P$ problem $\left(Q P_{c, \infty}^{i}\right)$ generated by the SQP method in solving $(\hat{P})$ is infeasible.

Proof. If $\left(Q P_{c, \infty}^{i}\right)$ is infeasible, then it follows that there exists no step $d$ such that:

$$
\begin{gathered}
\mathbf{f}^{(i)}+\nabla_{x, y} \mathbf{f}^{(i)^{T}} d \leq U-\epsilon \\
\mathbf{g}^{(i)}+\nabla_{x, y} \mathbf{g}^{(i)^{T}} d \leq 0
\end{gathered}
$$

Since (A.2) is an outer approximation of the nonlinear constraints of $(\hat{P})$ and (A.1) underestimates $\mathbf{f}(x, y)$, it follows that there exists no point $(\hat{x}, \hat{y}) \in X \times \hat{Y}$ such that:

$$
\mathbf{f}(\hat{x}, \hat{y}) \leq U-\epsilon
$$

Thus, any lower bound on $\mathbf{f}(x, y)$ at the present node has to be larger than $U-\epsilon$. So, within the tolerance $\epsilon$, $\mathbf{f}(x, y) \geq U$ and the fathoming rule FR3 holds.

The finiteness of integer variables implies that the branch-and-bound tree is finite. Thus, the algorithm must terminate after visiting a finite number of nodes. The nodes are only fathomed if they are infeasible, integer feasible or if the lower bounding of Lemma 1 holds. Thus, an optimal node must eventually be solved and the convergence follows from the convergence of the trust-region SQP method.

\section{Appendix B. Proof of Theorem 2}

Proposition 1. For the fixed retailer price vector $\mathcal{P}$, the reaction strategy $S_{k} \in \mathscr{S}_{k}\left(S_{R}\right)$ of each consumer $k \in \mathcal{K}$ is singleton, where $\mathscr{S}_{k}\left(S_{R}\right)$ is the reaction strategy set of consumer $k$ when the strategy of the retailer is fixed at $S_{R}$.

Proof. According to Theorem 1, the proposed solution for solving each consumer problem (28) with constant $\mathcal{P}^{h}, \forall h \in \mathcal{H}$, converges to unique optimal solution. This unique solution is the consumer reaction strategy, which completes the proof. 
So, based on Proposition 1, the optimization problem (41) can be rewritten as follows:

$$
\begin{array}{r}
\max _{S_{R} \in \mathscr{S}_{R}} U_{R}\left(S_{R}, \prod_{k=1}^{K} \mathscr{S}_{k}\left(S_{R}\right)\right) \\
\text { s.t., } S_{k} \in \underset{S_{k} \in \mathscr{S}_{k}}{\operatorname{argmax}} U_{k}\left(S_{k}, S_{R}\right), \forall k \in \mathcal{K}
\end{array}
$$

From the retailer point of view, problem (B.1) can be treated as a mathematical program with an implicitly defined constraint region given by the consumers' decision model [40]. So, the solution of this problem achieves the maximum benefit for the retailer (satisfies condition (40)) as well as capturing unique strategy of each consumer responding to the optimal prices. Moreover, from Theorem 1 we know that the consumers' strategies are optimal (satisfies condition (39)), which completes the proof of Theorem 2.

\section{Appendix C. Proof of Theorem 3}

First, we convert self-constraint objective function (36) into non-constraint version by adding penalty term as follows:

$$
\begin{aligned}
\varphi_{k, a}^{p}\left(\overline{\boldsymbol{V}}_{k}, \boldsymbol{x}_{k, a}, \boldsymbol{\omega}_{k, a}\right) & =\boldsymbol{v}_{k, a} \boldsymbol{x}_{k, a}^{\top}-w_{k, a} \cdot U_{k, a}\left(\boldsymbol{x}_{k, a}, \boldsymbol{\omega}_{k, a}\right) \\
& -\eta_{1}\left(\boldsymbol{x}_{k, a}-\boldsymbol{x}_{k, a}^{\max }\right)+\eta_{2}\left(\boldsymbol{x}_{k, a}-\boldsymbol{x}_{k, a}^{\min }\right)
\end{aligned}
$$

where $\eta_{1}, \eta_{2}$ are Lagrange multipliers [29]. We add the quantity ${ }^{9} \sum_{b \neq a} \varphi_{k, b}\left(\overline{\boldsymbol{V}}_{k}, \boldsymbol{x}_{k, b}, \boldsymbol{\omega}_{k, b}\right)$ to each appliance objective function (C.1) resulting in the following equal objective function for each appliance $a \in \mathcal{A}_{k}$ :

$$
\begin{gathered}
\boldsymbol{\varphi}\left(\overline{\boldsymbol{V}}_{k}, \boldsymbol{x}_{k, 1}, \cdots, \boldsymbol{x}_{k, A_{k}}, \boldsymbol{\omega}_{k, 1}, \cdots, \boldsymbol{\omega}_{k, A_{k}}\right)= \\
\sum_{b=1}^{A_{k}}\left(\boldsymbol{v}_{k, b} \boldsymbol{x}_{k, b}^{\top}-w_{k, b} \cdot U_{k, b}\left(\boldsymbol{x}_{k, b}, \boldsymbol{\omega}_{k, b}\right)\right. \\
\left.\quad-\boldsymbol{\eta}_{1}\left(\boldsymbol{x}_{k, a}-\boldsymbol{x}_{k, a}^{\max }\right)+\boldsymbol{\eta}_{2}\left(\boldsymbol{x}_{k, a}-\boldsymbol{x}_{k, a}^{\min }\right)\right)
\end{gathered}
$$

To prove the socially stable outcome of the game of stage 2 , we just need to prove the existence of a solution that maximizes (C.2). Let denote the available energy capacity coupled-constraint with $\sum_{a=1}^{A_{k}} x_{k, a} \leq E_{k, a}^{c a p}$, where $E_{k, a}^{c a p}$ is the maximum available energy and $x_{k, a}=\sum_{h=1}^{H} x_{k, a}^{h}$. Using the method of Lagrange multipliers [45], the Karush-Kuhn-Tucker (KKT) conditions for the ath appliance GNE problem is given by:

$$
\begin{aligned}
& \nabla_{x_{k, a}} \varphi_{k, a}^{p}\left(\overline{\boldsymbol{V}}_{k}, \boldsymbol{x}_{k, a}, \boldsymbol{\omega}_{k, a}\right) \\
&+\nabla_{x_{k, a}}\left(\sum_{a=1}^{A_{k}} x_{k, a}-E_{k, a}^{c a p}\right) \lambda_{k, a}=0 \\
& \lambda_{k, a}\left(\sum_{a=1}^{A_{k}} x_{k, a}-E_{k, a}^{c a p}\right)=0, \lambda_{k, a} \geq 0
\end{aligned}
$$

where $\lambda_{k, a}$ is the Lagrange multiplier for appliance $a$. As for a fixed price $\overline{\boldsymbol{V}}_{k}$, the followers' game admits a jointly convex GNE problem, the solution of the GNE problem with coupled constraint can be found via a variational inequality VI $(\mathbf{X}, \mathbf{F}(\mathbf{x}))[46]$. This essentially reduces to determining a vector $z^{*} \in \mathbf{X} \in \mathbb{R}^{n}$, such that

\footnotetext{
${ }^{9}$ Note that this quantity is treated as a constant quantity and does not change the problem solution.
} 
$\left\langle\mathbf{F}\left(\mathbf{z}^{*}\right), \mathbf{z}-\mathbf{z}^{*}\right\rangle \geq 0, \forall z \in \mathbf{X}$, where $\mathbf{X}$ is the set in the definition of joint convexity and $\mathbf{F}(\mathbf{x})=\left(\nabla_{x} \varphi_{k, a}^{p}(\mathbf{x})\right)_{a=1}^{A_{k}}$ [47]. The solution of $V I(\mathbf{X}, \mathbf{F}(\mathbf{x}))$ is a variational equilibrium $(\mathrm{VE})$. Now the KKT conditions are:

$$
\begin{aligned}
& \mathbf{F}(\mathbf{x})+\lambda_{k} \cdot \nabla_{x_{k, a}}\left(\sum_{a=1}^{A_{k}} x_{k, a}-E_{k, a}^{c a p}\right)=0 \\
& \lambda_{k} \cdot\left(\sum_{a=1}^{A_{k}} x_{k, a}-E_{k, a}^{c a p}\right)=0, \quad \lambda_{k} \geq 0
\end{aligned}
$$

where the subscript $a$ in Lagrange multiplier $\lambda_{k}$ is dropped due to the fact that the solution of a jointly convex GNE problem is a VE if and only if the coupled constraint has the same multiplier for all the players [46]. By the definition, $\mathbf{F}(\mathbf{x})$ can be denoted in matrix form as:

$$
\mathbf{F}=\left[\begin{array}{c}
\mathbf{f}_{k, 1} \\
\mathbf{f}_{k, 2} \\
\vdots \\
\mathbf{f}_{k, A_{k}}
\end{array}\right]
$$

with

$$
\mathbf{f}_{k, a}=\boldsymbol{v}_{k, a}+w_{k, a} \cdot\left(\left(\boldsymbol{\omega}_{k, a} \cdot \frac{1}{-m_{k, a}}\right) \cdot e^{\boldsymbol{\omega}_{k, a}\left(1-\left(\boldsymbol{x}_{k, a} / m_{k, a}\right)\right)}\right)-\boldsymbol{\eta}_{1}+\boldsymbol{\eta}_{2}
$$

The Jacobian matrix of $\mathbf{F}$ becomes:

$$
\mathbf{J F}=\left[\begin{array}{cccc}
\hat{\mathbf{f}}_{k, 1} & 0 & \cdots & 0 \\
0 & \hat{\mathbf{f}}_{k, 2} & \vdots & 0 \\
\vdots & 0 & \vdots & \vdots \\
0 & 0 & \cdots & \dot{\mathbf{f}}_{k, A_{k}}
\end{array}\right]
$$

with

$$
\dot{\mathbf{f}}_{k, a}=w_{k, a} \cdot\left(\left(\boldsymbol{\omega}_{k, a} \cdot \frac{1}{-m_{k, a}}\right)^{2} \cdot e^{\boldsymbol{\omega}_{k, a}\left(1-\left(\boldsymbol{x}_{k, a} / m_{k, a}\right)\right)}\right)
$$

Since $w_{k, a}, m_{k, a}>0, \omega_{k, a}^{h}, x_{k, a}^{h} \geq 0, \forall h \in \mathbb{H}$, and $x_{k, a}^{h}$ is a bounded quantity, all the diagonal elements of diagonal matrix $\mathbf{J F}$ are positive. Hence, $\mathbf{J F}$ is positive definite on $\mathbf{X}$, and therefore, $\mathbf{F}$ is strictly monotone. Thus, the GNE problem admits a unique global VE solution. Because of jointly convex nature of the GNE problem, the $\mathrm{VE}$ is the unique global minimizer of (C.2) [46]. So, we can claim that the proposed method, in which the ECS sets its optimal virtual price in response to the VE demands of the ECS's appliances, represents the socially unique optimal solution of Stackelberg game, which completes the proof.

\section{References}

[1] A. Q. Huang, M. L. Crow, G. T. Heydt, J. P. Zheng, and S. J. Dale, "The future renewable electric energy delivery and management (freedm) system: The energy internet," Proceedings of the IEEE, vol. 99, no. 1, pp. 133-148, Jan 2011.

[2] N. Lu, "An evaluation of the hvac load potential for providing load balancing service," IEEE Transactions on Smart Grid, vol. 3, no. 3, pp. 1263-1270, Sept 2012. 
[3] M. Albadi and E. El-Saadany, "A summary of demand response in electricity markets," Electric Power Systems Research, vol. 78, no. 11, pp. 1989 - 1996, 2008.

[4] D. Angeli and P. A. Kountouriotis, "A stochastic approach to dynamic-demand refrigerator control," IEEE Transactions on Control Systems Technology, vol. 20, no. 3, pp. 581-592, May 2012.

[5] Z. Zhu, S. Lambotharan, W. H. Chin, and Z. Fan, "A game theoretic optimization framework for home demand management incorporating local energy resources," IEEE Transactions on Industrial Informatics, vol. 11, no. 2, pp. 353-362, April 2015.

[6] S. Althaher, P. Mancarella, and J. Mutale, "Automated demand response from home energy management system under dynamic pricing and power and comfort constraints," IEEE Transactions on Smart Grid, vol. 6, no. 4, pp. 1874-1883, July 2015.

[7] S. H. Tindemans, V. Trovato, and G. Strbac, "Decentralized control of thermostatic loads for flexible demand response," IEEE Transactions on Control Systems Technology, vol. 23, no. 5, pp. 1685-1700, Sept 2015.

[8] H. T. Roh and J. W. Lee, "Residential demand response scheduling with multiclass appliances in the smart grid," IEEE Transactions on Smart Grid, vol. 7, no. 1, pp. 94-104, Jan 2016.

[9] F. Ye, Y. Qian, and R. Q. Hu, "A real-time information based demand-side management system in smart grid," IEEE Transactions on Parallel and Distributed Systems, vol. 27, no. 2, pp. 329-339, Feb 2016.

[10] M. Yu and S. H. Hong, "A real-time demand-response algorithm for smart grids: A stackelberg game approach," IEEE Transactions on Smart Grid, vol. 7, no. 2, pp. 879-888, March 2016.

[11] J. Hu, J. Cao, T. Yong, J. M. Guerrero, M. Z. Q. Chen, and Y. Li, "Demand response load following of source and load systems," IEEE Transactions on Control Systems Technology, vol. PP, no. 99, pp. 1-13, 2016.

[12] D. Croce, F. Giuliano, I. Tinnirello, A. Galatioto, M. Bonomolo, M. Beccali, and G. Zizzo, "Overgrid: A fully distributed demand response architecture based on overlay networks," IEEE Transactions on Automation Science and Engineering, vol. 14, no. 2, pp. 471-481, April 2017.

[13] N. Liu, M. Cheng, X. Yu, J. Zhong, and J. Lei, "Energy-sharing provider for pv prosumer clusters: A hybrid approach using stochastic programming and stackelberg game," IEEE Transactions on Industrial Electronics, vol. 65 , no. 8, pp. 6740-6750, Aug 2018.

[14] L. Xiao, X. Xiao, C. Dai, M. Pengy, L. Wang, and H. V. Poor, "Reinforcement learning-based energy trading for microgrids," arXiv preprint arXiv:1801.06285, 2018.

[15] A. J. Conejo, J. M. Morales, and L. Baringo, "Real-time demand response model," IEEE Transactions on Smart Grid, vol. 1, no. 3, pp. 236-242, Dec 2010.

[16] A. H. Mohsenian-Rad and A. Leon-Garcia, "Optimal residential load control with price prediction in real-time electricity pricing environments," IEEE Transactions on Smart Grid, vol. 1, no. 2, pp. 120-133, Sept 2010. 
[17] P. Samadi, H. Mohsenian-Rad, R. Schober, and V. W. S. Wong, "Advanced demand side management for the future smart grid using mechanism design," IEEE Transactions on Smart Grid, vol. 3, no. 3, pp. 1170-1180, Sept 2012.

[18] S. Maharjan, Q. Zhu, Y. Zhang, S. Gjessing, and T. Basar, "Dependable demand response management in the smart grid: A stackelberg game approach," IEEE Transactions on Smart Grid, vol. 4, no. 1, pp. 120-132, March 2013.

[19] M. Latifi, A. Khalili, A. Rastegarnia, and S. Sanei, "Fully distributed demand response using the adaptive diffusionstackelberg algorithm," IEEE Transactions on Industrial Informatics, vol. 13, no. 5, pp. 2291-2301, Oct 2017 .

[20] H. ALSalloum, A. ELMasri, L. Merghem-Boulahia, and R. Rahim, "Demand side management in smart grids: A stackelberg multi period multi provider game," in 2018 9th IFIP International Conference on New Technologies, Mobility and Security (NTMS), Feb 2018, pp. 1-5.

[21] C. Lee, L. Park, and S. Cho, "Light-weight stackelberg game theoretic demand response scheme for massive smart manufacturing systems," IEEE Access, vol. 6, pp. 23 316-23 324, 2018.

[22] P. Shinde and K. Shanti Swarup, "Stackelberg game-based demand response in multiple utility environments for electric vehicle charging," IET Electrical Systems in Transportation, vol. 8, no. 3, pp. 167-174, 2018.

[23] C. G. Cassandras and S. Lafortune, Introduction to Discrete Event Systems. Secaucus, NJ, USA: SpringerVerlag New York, Inc., 2006.

[24] L. C. Totu, J. Leth, and R. Wisniewski, "Control for large scale demand response of thermostatic loads*," in 2013 American Control Conference, June 2013, pp. 5023-5028.

[25] M. Stadler, W. Krause, M. Sonnenschein, and U. Vogel, "Modelling and evaluation of control schemes for enhancing load shift of electricity demand for cooling devices," Environmental Modelling and Software, vol. 24, no. 2, pp. $285-295,2009$.

[26] G. Sideratos and N. D. Hatziargyriou, "An advanced statistical method for wind power forecasting," IEEE Transactions on Power Systems, vol. 22, no. 1, pp. 258-265, Feb 2007.

[27] E. Lorenz, J. Hurka, D. Heinemann, and H. G. Beyer, "Irradiance forecasting for the power prediction of gridconnected photovoltaic systems," IEEE Journal of Selected Topics in Applied Earth Observations and Remote Sensing, vol. 2, no. 1, pp. 2-10, March 2009.

[28] H. K. Nguyen, J. B. Song, and Z. Han, "Demand side management to reduce peak-to-average ratio using game theory in smart grid," in 2012 Proceedings IEEE INFOCOM Workshops, March 2012, pp. 91-96.

[29] S. Boyd and L. Vandenberghe, Convex Optimization. New York: Cambridge University Press, 2004.

[30] M. Fahrioglu and F. L. Alvarado, "Using utility information to calibrate customer demand management behavior models," IEEE Transactions on Power Systems, vol. 16, no. 2, pp. 317-322, May 2001. 
[31] S. Leyffer, "Integrating sqp and branch-and-bound for mixed integer nonlinear programming," Computational Optimization and Applications, vol. 18, no. 3, pp. 295-309, 2001.

[32] J. Clausen, "Branch and bound algorithms-principles and examples," Parallel Computing in Optimization, pp. 239-267, 1997.

[33] R. Fletcher and S. Leyffer, "Nonlinear programming without a penalty function," Mathematical Programming, vol. 91, no. 2, pp. 239-269, 2002.

[34] P. T. Boggs and J. W. Tolle, "Sequential quadratic programming," Acta Numerica, vol. 4, p. 151, 1995.

[35] B. Borchers and J. E. Mitchell, "An improved branch and bound algorithm for mixed integer nonlinear programs," Computers and Operations Research, vol. 21, pp. 359-367, 1994.

[36] A. H. Mohsenian-Rad, V. W. S. Wong, J. Jatskevich, R. Schober, and A. Leon-Garcia, "Autonomous demandside management based on game-theoretic energy consumption scheduling for the future smart grid," IEEE Transactions on Smart Grid, vol. 1, no. 3, pp. 320-331, Dec 2010.

[37] H. M. Soliman and A. Leon-Garcia, "Game-theoretic demand-side management with storage devices for the future smart grid," IEEE Transactions on Smart Grid, vol. 5, no. 3, pp. 1475-1485, May 2014.

[38] P. Varutti, B. Kern, T. Faulwasser, and R. Findeisen, "Event-based model predictive control for networked control systems," in Proceedings of the 48h IEEE Conference on Decision and Control (CDC) held jointly with 2009 28th Chinese Control Conference, Dec 2009, pp. 567-572.

[39] M. S. Bazaraa, H. D. Sherali, and C. M. Shetty, Nonlinear programming : theory and algorithms, ser. WileyInterscience series in discrete mathematics and optimization. New York (N.Y.), Chichester, Brisbane: John Wiley \& Sons, 1993.

[40] J. F. Bard, Practical bilevel optimization: algorithms and applications. Springer Science \& Business Media, 2013, vol. 30 .

[41] T. Başar and G. J. Olsder, Dynamic noncooperative game theory. SIAM, 1998.

[42] M. J. Osborne and A. Rubinstein, A course in game theory. The MIT press, 1994.

[43] M. Simaan and J. Cruz, "A stackelberg solution for games with many players," IEEE Transactions on Automatic Control, vol. 18, no. 3, pp. 322-324, Jun 1973.

[44] Pennsylvania-New Jersey-Maryland Interconnection (PJM) electricity market, 2019. [Online]. Available: https://www.pjm.com/markets-and-operations/etools/data-miner-2/data-availability.aspx

[45] D. P. Bertsekas and D. P. Bertsekas, Nonlinear Programming, 2nd ed. Athena Scientific, Sep. 1999.

[46] F. Facchinei and C. Kanzow, "Generalized nash equilibrium problems," 4OR, vol. 5, no. 3, pp. 173-210, Sep 2007. 
[47] D. Kinderlehrer and G. Stampacchia, An Introduction to Variational Inequalities and Their Applications, ser. Pure and Applied Mathematics. Elsevier Science, 1980. 